\title{
Genus stabilization for the components of moduli spaces of curves with symmetries
}

\author{
Fabrizio Catanese, Michael Lönne and Fabio Perroni
}

\begin{abstract}
In a previous paper (Groups, Geometry, and Dynamics, 2015), we introduced a new homological invariant $\varepsilon$ for the faithful action of a finite group $G$ on an algebraic curve. We show here that the moduli space of curves admitting a faithful action of a finite group $G$ with a fixed homological invariant $\varepsilon$, if the genus $g^{\prime}$ of the quotient curve satisfies $g^{\prime} \gg 0$, is irreducible (and non-empty if and only if the class satisfies the 'admissibility' condition). We achieve this by showing that the stable equivalence classes of Hurwitz generating systems are in bijection with the admissible classes $\varepsilon$.
\end{abstract}

\section{Introduction}

The main purpose of this article is the determination of the irreducible components of the moduli spaces of curves admitting a given symmetry group $G$. As shown in [CLP11] and [CLP15], this is an important step for understanding the structure of the locus of curves with automorphisms (see [Cor87, Cor08, Cat12]) inside the moduli space $\mathfrak{M}_{g}$ of curves of genus $g$ (see [DM69, Ful69]). Our main result, Theorem 3.4, states that those components corresponding to a fixed branching data of the $G$-cover are indexed via the $\varepsilon$-invariant (see Section 2) by a certain quotient of the second homology group of $\mathrm{G}$, when the genus $g^{\prime}$ of the quotient curve $C^{\prime}:=C / G$ is large enough. We view this result as saying that the 0th Betti numbers of these moduli spaces stabilize, and consider it as an initial step towards the investigation of their homological stabilization.

Recall that an extensive amount of literature has been devoted to the related case of Hurwitz spaces, namely, moduli spaces of ramified coverings $\pi: C \rightarrow B$ with $B$ a fixed curve of genus $g^{\prime}=0$ or higher (see [Cle73, Hur91, Klu88, Edm82, Edm83, BF86, BC97, Waj96, GHS02, Kan06, Kan05, Sia09, Vet06, Vet07, Vet08, CLP11]).

Let us first introduce the framework into which the stabilization theorem proven in this paper fits. Let $g>1$ be a positive integer, and let $C$ be a projective curve of genus $g$. Consider a subgroup $G$ of the group of automorphisms of $C$, and let $\gamma \in G \leqslant \operatorname{Aut}(C)$; then, since $C$ is

Received 17 January 2014, accepted in final form 19 April 2015.

2010 Mathematics Subject Classification 14H15, 14H10, 14H30, 14H37, 20J06, 30F60, 55M35, 57M12, 57S05.

Keywords: Algebraic curves with automorphisms, moduli spaces, mapping class group, Teichmüller space, Hurwitz monodromy vector, homological invariant, genus stabilization.

This journal is (C) Foundation Compositio Mathematica 2016. This article is distributed with Open Access under the terms of the Creative Commons Attribution Non-Commercial License, which permits non-commercial reuse, distribution, and reproduction in any medium, provided that the original work is properly cited. For commercial re-use, please contact the Foundation Compositio Mathematica.

The present work took place in the realm of the DFG Forschergruppe 790 "Classification of algebraic surfaces and compact complex manifolds". A revision was worked out in the realm of the ERC-2013-Advanced Grant - 340258TADMICAMT. The third author was partially supported by the FRA grants 2013 and 2015 of the University of Trieste, the PRIN "Geometria delle Varietà Algebriche", and a grant of GNSAGA of INDAM. 


\section{F. Catanese, M. Lönne and F. Perroni}

a $K\left(\Pi_{g}, 1\right)$ space, the homotopy class of $\gamma: C \rightarrow C$ is determined by

$$
\pi_{1}(\gamma): \pi_{1}\left(C, y_{0}\right) \rightarrow \pi_{1}\left(C, \gamma\left(y_{0}\right)\right)
$$

and, actually, the topological type of the action is completely determined by its action on the fundamental group, up to inner automorphisms. Hence, we get a group homomorphism

$$
\rho: \quad G \rightarrow \operatorname{Aut}\left(\Pi_{g}\right) / \operatorname{Inn}\left(\Pi_{g}\right)=\operatorname{Out}\left(\Pi_{g}\right) .
$$

This homomorphism $\rho$ is injective by Lefschetz' lemma $\left(\gamma \underset{h}{\sim} \operatorname{id}_{C} \Rightarrow \gamma=\operatorname{id}_{C}\right)$ asserting that, for $g \geqslant 2$, only the identity transformation is homotopic to the identity. Moreover, since holomorphic maps are orientation preserving, we actually have

$$
\rho: G \rightarrow \operatorname{Out}^{+}\left(\Pi_{g}\right)=\operatorname{Map}_{g} \cong \operatorname{Diff}^{+}(C) / \operatorname{Diff}^{0}(C),
$$

and indeed $\rho$ determines the differentiable type of the action.

When we speak of the moduli space of curves admitting an action by a finite group $G$ of a fixed topological type, we fix $\rho(G)$ up to the action of $\operatorname{Aut}(G)$ on the source and the adjoint action of the mapping class group $\mathrm{Map}_{g}$ on the target. The class of $\rho(G)$ thus obtained is the 'unmarked topological type' of the action. In order to be more precise, let us recall the description of the moduli spaces of curves via Teichmüller theory.

Fix $M$ to be the underlying oriented differentiable manifold of $C$, and let $\mathcal{C} S(M)$ be the space of complex structures on $M$ compatible with the given orientation; then the moduli space of curves $\mathfrak{M}_{g}$ and Teichmüller space $\mathcal{T}_{g}$ are defined as

$\mathfrak{M}_{g}:=\mathcal{C} S(M) / \operatorname{Diff}^{+}(M) \quad$ and $\quad \mathcal{T}_{g}:=\mathcal{C} S(M) / \operatorname{Diff}^{0}(M), \quad$ so that we have $\quad \mathfrak{M}_{g}=\mathcal{T}_{g} / \operatorname{Map}_{g}$.

Teichmüller's theorem says that $\mathcal{T}_{g} \subset \mathbb{C}^{3 g-3}$ is an open subset diffeomorphic to a ball. Moreover, $\operatorname{Map}_{g}$ acts properly discontinuously on $\mathcal{T}_{g}$, but not freely. As a corollary, the rational cohomology of the moduli space is calculated by group cohomology: $H^{*}\left(\mathfrak{M}_{g}, \mathbb{Q}\right) \cong H^{*}\left(\operatorname{Map}_{g}, \mathbb{Q}\right)$.

Harer [Har85] showed that these cohomology groups stabilize with $g$.

Definition 1.1. The manifold $\mathcal{T}_{g, \rho}:=\mathcal{T}_{g}^{\rho(G)}$ is the fixed-point locus of $\rho(G)$ in $\mathcal{T}_{g}$.

The first author proved an analogue of Teichmüller's theorem, namely that $\mathcal{T}_{g, \rho}$ is diffeomorphic to a ball [Cat00]. (For the study of deformations of compact complex manifolds with automorphisms we refer to [Cat88].) Hence we get other subvarieties of the moduli space according to the following definition.

Definition 1.2. The subset $\mathfrak{M}_{g, \rho}$ is the image of $\mathcal{T}_{g, \rho}$ in $\mathfrak{M}_{g}$. It is an irreducible Zariski closed subvariety, as shown in [Cat12] and stated in [CLP15].

The marked moduli space $\mathfrak{M M}_{g, \rho}$ is the quotient of $\mathcal{T}_{g, \rho}$ by the centralizer of $\rho(G) \leqslant \operatorname{Map}_{g}$ (the action of $G$ is given together with a marking of $G$; we do not allow changing the given action of $G$ via an automorphism of $G$ ).

Observe that the closed irreducible subvariety $\mathfrak{M}_{g, \rho}$ depends only upon the unmarked topological type of the $G$-action.

The above description of the space of curves admitting a given topological action of a group $G$ is quite nice, but not completely explicit. One can make everything more explicit via a more precise understanding obtained through geometry; in this way one can find discrete invariants of the topological type of the action. 


\section{Genus STABILIZATION FOR MODULI OF CURVE-SYMMETRIES}

Consider the quotient curve $C^{\prime}:=C / G$, let $g^{\prime}:=g\left(C^{\prime}\right)$ be its genus, let $\mathcal{B}=\left\{y_{1}, \ldots, y_{d}\right\}$ be the branch locus, and let $m_{i}$ be the branching multiplicity of $y_{i}$. Observe that the numbers $g^{\prime}, d$, $m_{1}, \ldots, m_{d}$ are constant in each irreducible variety $\mathfrak{M}_{g, \rho}$; they form the 'primary numerical type' of the cover $C \rightarrow C^{\prime}$.

The branched covering $C \rightarrow C^{\prime}$ is determined (by virtue of Riemann's existence theorem) by the monodromy $\mu: \pi_{1}\left(C^{\prime} \backslash \mathcal{B}, y_{0}\right) \rightarrow G$. We have

$$
\pi_{1}\left(C^{\prime} \backslash \mathcal{B}, y_{0}\right) \cong \Pi_{g^{\prime}, d}:=\left\langle\gamma_{1}, \ldots, \gamma_{d}, \alpha_{1}, \beta_{1}, \ldots, \alpha_{g^{\prime}}, \beta_{g^{\prime}} \mid \prod_{i=1}^{d} \gamma_{i} \prod_{j=1}^{g^{\prime}}\left[\alpha_{j}, \beta_{j}\right]=1\right\rangle .
$$

Therefore the datum of $\mu$ is equivalent to the datum of a Hurwitz generating system, that is, of a Hurwitz-vector

$$
v:=\left(c_{1}, \ldots, c_{d}, a_{1}, b_{1}, \ldots, a_{g^{\prime}}, b_{g^{\prime}}\right) \in G^{d+2 g^{\prime}}
$$

such that

(i) the group $G$ is generated by the entries $c_{1}, \ldots, c_{d}, a_{1}, b_{1}, \ldots, a_{g^{\prime}}, b_{g^{\prime}}$;

(ii) $c_{i} \neq 1_{G}$ for all $i$;

(iii) we have

$$
\prod_{i=1}^{d} c_{i} \prod_{j=1}^{g^{\prime}}\left[a_{j}, b_{j}\right]=1
$$

Riemann's existence theorem shows that the unmarked topological types with primary numerical types of the form $g^{\prime}, d, m_{1}, \ldots, m_{d}$ with fixed $g^{\prime}$ and $d$ are in bijection with the points of the orbit space

$$
\left(\operatorname{Epi}\left(\Pi_{g^{\prime}, d}, G\right) / \operatorname{Aut}(G)\right) / \operatorname{Map}\left(g^{\prime}, d\right)
$$

(here 'Epi' stands for 'set of epimorphisms').

\section{Relation between the two approaches through the orbifold fundamental group $\pi_{1}^{\text {orb }}$}

For more details concerning the orbifold fundamental group and its applications in this context we refer to [Cat08].

Let $X$ be a 'good' topological space, and let $\tilde{X}$ be the universal cover of $X$. Assume that $G$ is a finite group acting effectively on $X$. Then $G$ can be lifted to a discontinuous group $\tilde{G}$ of homeomorphisms of $\tilde{X}$ in such a way that the quotients $\tilde{X} / \tilde{G}$ and $X / G$ are homeomorphic. The group $\tilde{G}$ is called the orbifold fundamental group of the $G$-action and is an extension of $\pi_{1}(X)$ by $G$; that is, we have a short exact sequence

$$
1 \rightarrow \pi_{1}(X) \rightarrow \tilde{G}=: \pi_{1}^{\text {orb }}(X / G) \rightarrow G \rightarrow 1 .
$$

In our situation, $X=C$ and its universal cover is the upper half-plane $\tilde{X}=\mathbb{H}$; hence $\pi_{1}^{\text {orb }}(X / G)$ is a Fuchsian group. We have

$$
C=\mathbb{H} / \Pi_{g}, \quad C^{\prime}=\mathbb{H} / \pi_{1}^{\text {orb }}, \quad \pi_{1}^{\text {orb }}=\Pi_{g^{\prime}, d} /\left\langle\left\langle\gamma_{1}^{m_{1}}, \ldots, \gamma_{d}^{m_{d}}\right\rangle\right\rangle,
$$

where, as usual, $\left\langle\left\langle a_{1}, \ldots, a_{d}\right\rangle\right\rangle$ denotes the subgroup normally generated by the elements $a_{1}, \ldots, a_{d}$.

The above exact sequence yields, via conjugation acting on the normal subgroup $\pi_{1}(X) \cong \Pi_{g}$, a homomorphism $\rho: G \rightarrow \operatorname{Out}\left(\Pi_{g}\right)$.

Fix now $d$ points $y_{1}, \ldots, y_{d}$ on the oriented differentiable manifold $M^{\prime}$ underlying $C^{\prime}$, and set 


\section{F. Catanese, M. Lönne and F. Perroni}

$\mathcal{B}:=\left\{y_{1}, \ldots, y_{d}\right\}$. One can then define the $d$-marked Teichmüller space as the quotient

$$
\mathcal{T}_{g^{\prime}, d}:=\mathcal{C} S\left(M^{\prime}\right) / \operatorname{Diff}^{0}\left(M^{\prime}, \mathcal{B}\right) .
$$

We thus have an unramified covering map of connected spaces $\mathcal{T}_{g^{\prime}, d} \rightarrow \mathcal{T}_{g, \rho}$; since $\mathcal{T}_{g, \rho}$ is a ball, this map is a homeomorphism (hence, in particular, the topological type $\rho$ also determines the monodromy $\mu$, as can be proven directly).

We can now describe the numerical and homological invariants of $\rho$ (equivalently, of $\mu$ or of $v$ ). The first numerical invariant is called (by different authors) the branching numerical function, Nielsen class, or $\nu$-type of $\rho$.

Definition 1.3. Let $\operatorname{Conj}(G)$ be the set of (non-trivial) conjugacy classes in the group $G$ : then we let $\nu: \operatorname{Conj}(G) \rightarrow \mathbb{N}, \nu(\mathcal{C}):=\left|\left\{i \mid c_{i} \in \mathcal{C}\right\}\right|$, be the function which counts how many local monodromy elements (these are defined only up to conjugation) are in a fixed conjugacy class.

This invariant was first introduced by Nielsen [Nie37], who proved that $\nu$ determines $\rho$ if $G$ is cyclic.

The (semi-)classical homological invariant is instead defined as follows. Let $H:=\left\langle\left\langle c_{1}, \ldots, c_{d}\right\rangle\right\rangle$ be the subgroup normally generated by the local monodromies (local generators of the isotropy subgroups). Consider the quotient group $G^{\prime \prime}:=G / H$. The covering $C \rightarrow C^{\prime}=C / G$ factors through $C^{\prime \prime}=C / H \rightarrow C^{\prime}$, which is unramified and with group $G^{\prime \prime}$. The monodromy $\mu^{\prime \prime}: \Pi_{g^{\prime}} \rightarrow G^{\prime \prime}$ corresponds to a homotopy class of a continuous map $m^{\prime \prime}: C^{\prime} \rightarrow \mathrm{K}\left(G^{\prime \prime}, 1\right)$. Passing to homology, we have

$$
H_{2}\left(m^{\prime \prime}\right)=H_{2}\left(\mu^{\prime \prime}\right): H_{2}\left(\Pi_{g^{\prime}}, \mathbb{Z}\right)=H_{2}\left(C^{\prime}, \mathbb{Z}\right) \rightarrow H_{2}\left(G^{\prime \prime}, \mathbb{Z}\right) .
$$

One then defines $h(v)$ as the image of the fundamental class of $C^{\prime}$, the generator $\left[C^{\prime}\right]$ of $H_{2}\left(C^{\prime}, \mathbb{Z}\right)$ determined by the complex orientation:

$$
h(v):=H_{2}\left(m^{\prime \prime}\right)\left[C^{\prime}\right] \in H_{2}\left(G^{\prime \prime}, \mathbb{Z}\right) .
$$

More generally, Edmonds [Edm82, Edm83] showed that $\nu$ and $h$ determine $\rho$ for $G$ abelian, and that if, moreover, $G$ is split-metacyclic and the action is free (that is, $G=G^{\prime \prime}$ ), then $h$ determines $\rho$.

In our recent paper [CLP15] we considered the case $G=D_{n}$ of the dihedral group of order $2 n$. After showing that in this case $(\nu, h)$ does not determine $\rho$, we introduced on these grounds a finer homological invariant $\varepsilon \in K_{\Gamma}$, where $\Gamma$ is the union of the conjugacy classes of the local monodromies $c_{i}$, and $K_{\Gamma}$ is a group constructed from the given group $G$ and the given subset $\Gamma$ (the definitions of $K_{\Gamma}$ and $\varepsilon$ are given in Section 2). The main result of [CLP15] was to prove that in the case of the dihedral group, our invariant $\varepsilon$ determines the class of $\rho$.

We do not recall here the definition of the group $K_{\Gamma}$ and of the invariant $\varepsilon(v)$, since Section 2 is devoted to these definitions and their topological interpretation (which was not contained in [CLP15]). It suffices here to observe that this new invariant encodes in particular all the classical numerical and homological invariants.

It would be a too nice and simple world if this invariant would do the job for any group. But this invariant, which in the unramified case coincides with the classical invariant in $H_{2}(G, \mathbb{Z})$, does not distinguish irreducible components in general (see [DT06]). It only does so if the genus $g^{\prime}$ of the quotient curve is large enough. The crucial point, as in Harer's theorem, is the concept of stabilization: 


\section{Genus STABILIZATION FOR MODULi OF CURVE-SyMmetries}

- direct stabilization: the monodromy $\mu$ is stabilized by extending it to a homomorphism of the fundamental group $\Pi_{g^{\prime}+1}$ into $G$ whose value on the last two generators $\alpha_{g^{\prime}+1}$ and $\beta_{g^{\prime}+1}$ is set to be $\mu\left(\alpha_{g^{\prime}+1}\right)=\mu\left(\beta_{g^{\prime}+1}\right)=1 \in G$; in other words, we add a handle to the quotient curve $C^{\prime}$, such that on it the monodromy is trivial;

- stabilization: this is defined as the equivalence relation generated by direct stabilization.

In the étale case it was shown that the homology invariant is a full 'stable' invariant.

Theorem 1.4 (Dunfield-Thurston). In the unramified case $(d=0)$, for $g^{\prime} \gg 0$, the set of unmarked topological types is in bijection with

$$
H_{2}(G, \mathbb{Z}) / \operatorname{Aut}(G)
$$

The proof of this theorem is based on the interpretation of second homology as bordism, and on Livingston's theorem [Liv85] showing that two unramified monodromies having the same homology class in $\mathrm{H}_{2}(G, \mathbb{Z})$ are stably equivalent. A very suggestive proof of Livingston's theorem, based on the concept of a relative Morse function with increasing Morse indices, is given in [DT06], while an algebraic proof is given by Zimmermann in [Zim87].

In the ramified case, the situation is much more complicated, and it turns out to be safer to rely on the algebraic technique of Zimmermann in order to set up a secure, even if technical, proof of the following main theorem.

TheOREm 1.5. For $g^{\prime} \gg 0$, the unmarked topological types are in bijection with the admissible classes via the map $\hat{\varepsilon}$ associated with the invariant $\varepsilon$.

In this theorem, the condition of admissibility is the simple translation of the condition that the product of the local monodromies $c_{1}, \ldots, c_{d}$ must be a product of commutators.

In a sequel to this paper we shall prove another stabilization, which we call branching stabilization, and which generalizes the following result (see [FV91]).

Theorem 1.6 (Conway-Parker). In the case $g^{\prime}=0$, let $G=F / R$, where $F$ is a free group, and assume that $H_{2}(G, \mathbb{Z}) \cong([F, F] \cap R) /[F, R]$ is generated by commutators. Then there is an integer $N$ such that if the numerical function $\nu$ takes values greater than or equal to $N$, then there is only one unmarked topological type with the given numerical function $\nu$.

We shall get an analogous result for any genus $g^{\prime}$, using our fuller homological invariant $\varepsilon$. In the course of proving branching stabilization, we shall also give a different proof of our genus stabilization result, using a variant of the semi-group and of the group introduced by Conway and Parker.

Finally, we want to mention the interesting question of determining the class of groups for which no stabilization is needed, thus extending to other groups the result we obtained for the dihedral groups.

The structure of the paper is the following. In Section 2 we first recall the definition of the $\varepsilon$-invariant from [CLP15]; then we give a topological interpretation by means of a certain relative homology group of the group $G$. This interpretation is not essential for the rest of the paper, but it gives a different and sometimes useful point of view. In Section 3 we state our main results, Theorems 3.4 and 3.5, and prove the former assuming the latter. Section 4 contains a proof of Theorem 3.5, which is an extension of Livingston's theorem [Liv85] to the ramified case. 


\section{F. Catanese, M. Lönne and F. Perroni}

\section{The $\varepsilon$-invariant}

In this section we first review the definition of the $\varepsilon$-invariant of Hurwitz vectors which was introduced in [CLP15, Section 3], then we give a topological interpretation of this invariant as a class in a certain relative homology group of $G$ modulo an equivalence relation. Although we do not explicitly use this topological interpretation in the proof of the main theorem, many of the technical results which we prove throughout the paper are just algebraic reformulations of simple geometrical statements whose understanding is easier using a topological view of the $\varepsilon$-invariant.

Let us first recall the following notions.

Definition 2.1. Let $G$ be a finite group, and let $g^{\prime}, d \in \mathbb{N}$. A $\left(g^{\prime}, d\right)$-Hurwitz vector in $G$ is an element $v$ of $G^{d+2 g^{\prime}}$, the $\left(d+2 g^{\prime}\right)$-fold Cartesian product of $G$. A $\left(g^{\prime}, d\right)$-Hurwitz vector in $G$ will also be denoted by

$$
v=\left(c_{1}, \ldots, c_{d} ; a_{1}, b_{1}, \ldots, a_{g^{\prime}}, b_{g^{\prime}}\right) .
$$

For any $i \in\left\{1, \ldots, d+2 g^{\prime}\right\}$, the $i$ th component $v_{i}$ of $v$ is defined as usual. The evaluation of $v$ is the element

$$
\mathrm{ev}(v):=\prod_{1}^{d} c_{j} \cdot \prod_{1}^{g^{\prime}}\left[a_{i}, b_{i}\right] \in G .
$$

A Hurwitz generating system of length $d+2 g^{\prime}$ in $G$ is a $\left(g^{\prime}, d\right)$-Hurwitz vector $v$ in $G$ such that the following conditions hold:

(i) The local monodromies $c_{i}$ are non-trivial; that is, $c_{i} \neq 1$ for all $i$.

(ii) The group $G$ is generated by the components $v_{i}$ of $v$.

(iii) $\operatorname{ev}(v)=1$.

We denote by $\operatorname{HS}\left(G ; g^{\prime}, d\right) \subset G^{d+2 g^{\prime}}$ the set of all Hurwitz generating systems in $G$ of type $\left(g^{\prime}, d\right)$ (hence of length $d+2 g^{\prime}$ ).

We refer to [Bir69] and [Waj99] for general results and definitions concerning mapping class groups.

Definition 2.2. For $d \geqslant 1$, denote by $\widetilde{\operatorname{Map}}\left(g^{\prime}, d\right)$ the group of isotopy classes of orientationpreserving diffeomorphisms of a curve $C^{\prime}$ of genus $g^{\prime}$ which leave invariant a set $\mathcal{B}$ of $d$ points and leave fixed a base point $y_{0}$.

For $d=0$, denote by $\widetilde{\operatorname{Map}}\left(g^{\prime}, 0\right)$ the group of isotopy classes of diffeomorphisms of a curve $C^{\prime}$ of genus $g^{\prime}$ which are the identity on a small disc $\Delta$ whose boundary circle $\partial \Delta$ contains $y_{0}$.

Remark 2.3. Recall that

$$
\Pi_{g^{\prime}, d}:=\left\langle\gamma_{1}, \ldots, \gamma_{d}, \alpha_{1}, \beta_{1}, \ldots, \alpha_{g^{\prime}}, \beta_{g^{\prime}} \mid \prod_{i=1}^{d} \gamma_{i} \prod_{j=1}^{g^{\prime}}\left[\alpha_{j}, \beta_{j}\right]=1\right\rangle
$$

is the quotient of the free group

$$
\mathbb{F}:=\left\langle x_{1}, \ldots, x_{d+2 g^{\prime}}\right\rangle
$$

under the morphism induced by

$$
x_{1} \mapsto \gamma_{1}, \ldots, x_{d} \mapsto \gamma_{d}, x_{d+1} \mapsto \alpha_{1}, x_{d+2} \mapsto \beta_{1}, \ldots, x_{d+2 g^{\prime}} \mapsto \beta_{g^{\prime}}
$$




\section{Genus STABILIZATION FOR MODUli OF CURVE-SYMmETRIES}

by the subgroup normally generated by

$$
E:=\prod_{i=1}^{d} x_{i} \prod_{j=1}^{g^{\prime}}\left[x_{d+2 j-1}, x_{d+2 j}\right] .
$$

Now, for $d \geqslant 1$, there is a well-determined isomorphism of $\Pi_{g^{\prime}, d}$ with the free group $\mathbb{F}^{\prime}:=$ $\left\langle x_{2}, \ldots, x_{d+2 g^{\prime}}\right\rangle$ :

$$
\begin{aligned}
\xi: \Pi_{g^{\prime}, d} & \rightarrow \mathbb{F}^{\prime} \\
\xi\left(\gamma_{1}\right) & =\left(\prod_{i=2}^{d} x_{i} \prod_{j=1}^{g^{\prime}}\left[x_{d+2 j-1}, x_{d+2 j}\right]\right)^{-1}, \\
\xi\left(\gamma_{2}\right) & =x_{2}, \ldots, \xi\left(\gamma_{d}\right)=x_{d}, \\
\xi\left(\alpha_{j}\right) & =x_{d+2 j-1}, \quad \xi\left(\beta_{j}\right)=x_{d+2 j} \quad \text { for } j=1, \ldots, g^{\prime} .
\end{aligned}
$$

In particular, there is a well-determined isomorphism of $\mathbb{F}$ with the free direct product $\mathbb{F}^{\prime} *\langle E\rangle \cong$ $\Pi_{g^{\prime}, d} *\langle E\rangle$, so that one gets a well-determined identification

$$
\operatorname{Aut}\left(\Pi_{g^{\prime}, d}\right) \cong\left\{\psi \in \operatorname{Aut}(\mathbb{F}) \mid \psi(E)=E, \psi\left(\mathbb{F}^{\prime}\right)=\mathbb{F}^{\prime}\right\} \subset\{\psi \in \operatorname{Aut}(\mathbb{F}) \mid \psi(E)=E\} .
$$

Notice that in $\mathbb{F}$, we have $\xi\left(\gamma_{1}\right)=E^{-1} x_{1}$.

Hence, for $d \geqslant 1$, the group $\widetilde{\operatorname{Map}}\left(g^{\prime}, d\right)$ acts on $\pi_{1}\left(C^{\prime} \backslash \mathcal{B}, y_{0}\right) \cong \Pi_{g^{\prime}, d}$. Via this homomorphism into $\operatorname{Aut}\left(\Pi_{g^{\prime}, d}\right)$ and the previous identification we therefore get a well-determined action of $\widetilde{\operatorname{Map}}\left(g^{\prime}, d\right)$ on the free group $\mathbb{F}$ which leaves fixed the word $E$. From this we obtain a welldetermined action of $\widetilde{\operatorname{Map}}\left(g^{\prime}, d\right)$ on the set of $\left(g^{\prime}, d\right)$-Hurwitz vectors $v$ in $G$, for any group $G$, not necessarily finite. Moreover, notice that, for any group homomorphism $f: G_{1} \rightarrow G_{2}$, the induced map $f^{d+2 g^{\prime}}: G_{1}^{d+2 g^{\prime}} \rightarrow G_{2}^{d+2 g^{\prime}}$ between the sets of Hurwitz vectors is $\widehat{\operatorname{Map}}\left(g^{\prime}, d\right)$-equivariant.

We shall now show that also for $d=0$ there is a well-determined homomorphism of $\widetilde{\operatorname{Map}}\left(g^{\prime}, 0\right)$ into $\{\psi \in \operatorname{Aut}(\mathbb{F}) \mid \psi(E)=E\}$ : this fact is important, because it shows that this action then leaves invariant the evaluation map of Hurwitz vectors. In fact, $\widetilde{\operatorname{Map}}\left(g^{\prime}, 0\right)$ acts on $C^{\prime} \backslash U$, where $U$ is the interior of the disc $\Delta$, whence on $\pi_{1}\left(C^{\prime} \backslash U, y_{0}\right) \cong \mathbb{F}$; the element $E$ corresponds to the circle $\partial \Delta$, giving the desired assertion.

There is an obvious surjection $\widetilde{\operatorname{Map}}\left(g^{\prime}, d\right) \rightarrow \operatorname{Map}\left(g^{\prime}, d\right)$, and one sees right away that the action of $\widetilde{\operatorname{Map}}\left(g^{\prime}, d\right)$ on the set of $\left(g^{\prime}, d\right)$-Hurwitz vectors $v$, and especially on the subset of Hurwitz generating systems, induces an action of $\operatorname{Map}\left(g^{\prime}, d\right)$ on the set of orbits of the set of Hurwitz generating systems in $G$ by the action of simultaneous conjugation by $G$. In particular, the orbit space

can also be seen as

$$
\left(\operatorname{Epi}\left(\Pi_{g^{\prime}, d}, G\right) / \operatorname{Aut}(G)\right) / \operatorname{Map}_{g^{\prime}, d}
$$

$$
\left(\operatorname{Epi}\left(\Pi_{g^{\prime}, d}, G\right) / \widetilde{\operatorname{Map}}\left(g^{\prime}, d\right)\right) / \operatorname{Aut}(G) .
$$

Definition 2.4. Let $G$ be a finite group. From now on, $F:=\langle\hat{g} \mid g \in G\rangle$ shall be the free group generated by the elements of $G$. Let $R \unlhd F$ be the normal subgroup of relations, so that $G=F / R$.

For any union of non-trivial conjugacy classes $\Gamma \subset G$, define

$$
\left.R_{\Gamma}:=\left\langle\left\langle[F, R], \hat{a} \hat{b} \hat{c}^{-1} \hat{b}^{-1}\right| \forall a, c \in \Gamma, b \in G \text { such that } b^{-1} a b=c\right\rangle\right\rangle,
$$

the minimal normal subgroup of $F$ generated by $[F, R]$ and $\hat{a} \hat{b} \hat{c}^{-1} \hat{b}^{-1}$, for any $a, c \in \Gamma, b \in G$ 


\section{F. Catanese, M. Lönne and F. Perroni}

such that $b^{-1} a b=c$, and set

$$
G_{\Gamma}:=F / R_{\Gamma} .
$$

The map $\hat{a} \mapsto a$ for $a \in G$ induces a group homomorphism $\alpha: G_{\Gamma} \rightarrow G$ whose kernel shall be denoted by $K_{\Gamma}:=\operatorname{Ker}(\alpha)$.

By [CLP15, Lemma 3.2], $K_{\Gamma}=R / R_{\Gamma}$ is contained in the centre of $G_{\Gamma}$.

Definition 2.5. Given a $\left(g^{\prime}, d\right)$-Hurwitz vector

$$
v=\left(c_{1}, \ldots, c_{d} ; a_{1}, b_{1}, \ldots, a_{g^{\prime}}, b_{g^{\prime}}\right)
$$

in $G$ (cf. Definition 2.1), its tautological lift $\hat{v}$ is the $\left(g^{\prime}, d\right)$-Hurwitz vector in $G_{\Gamma}$ defined by

$$
\hat{v}:=\left(\widehat{c_{1}}, \ldots, \widehat{c_{d}} ; \widehat{a_{1}}, \widehat{b_{1}}, \ldots, \widehat{a_{g^{\prime}}}, \widehat{b_{g^{\prime}}}\right) \text {, }
$$

whose components are the tautological lifts of the components of $v$.

Given a $\left(g^{\prime}, d\right)$-Hurwitz vector $v$ in $G$ with $c_{i} \neq 1$ for $i=1, \ldots d$, we denote by $\Gamma_{v}$ the union of all the conjugacy classes of $G$ containing at least one $c_{i}$.

For any Hurwitz generating system $v$ (or Hurwitz vector satisfying condition (iii) of Definition 2.1), set

$$
\varepsilon(v):=\prod_{1}^{d} \widehat{c_{j}} \cdot \prod_{1}^{g^{\prime}}\left[\widehat{a_{i}}, \widehat{b_{i}}\right] \in K_{\Gamma_{v}}
$$

to be the evaluation of the tautological lift $\hat{v}$ of $v$ in $G_{\Gamma_{v}}$ (cf. Definition 2.1).

We have already observed in Remark 2.3 that the evaluation map is invariant under the action of the mapping class group $\widehat{\operatorname{Map}}\left(g^{\prime}, d\right)$. In [CLP15, Proposition 3.6], using this invariance, we showed even more, namely that the evaluation map has the same value on the tautological lift $\hat{v}$ and on the tautological lift $\widehat{\varphi \cdot v}$ for any $\varphi \in \widehat{\operatorname{Map}}\left(g^{\prime}, d\right)$. For completeness we include here this result from [CLP15] with a complete proof.

Proposition 2.6. For any $g^{\prime}, d \in \mathbb{N}$, the map $\varepsilon$ is $\widetilde{\operatorname{Map}}\left(g^{\prime}, d\right)$-invariant.

Proof. Let $\varphi \in \widetilde{\operatorname{Map}}\left(g^{\prime}, d\right)$. We first consider the case where $\varphi$ is a Hurwitz move, that is, one of the standard generators of the braid group $\mathcal{B}_{d}$, a subgroup of $\widetilde{\operatorname{Map}}\left(g^{\prime}, d\right)$.

Without loss of generality we consider the first Hurwitz move $\sigma_{1}$ and assume $g^{\prime}=0$ and $d=2$. The action of $\sigma_{1}$ sends

$$
v=\left(c_{1}, c_{2}\right) \text { to } v^{\prime}=\left(c_{2}, c_{2}^{-1} c_{1} c_{2}\right) .
$$

Inside $G_{\Gamma_{v}}$ we have, thanks to the fact that $c_{1} \in \Gamma_{v}$ and that $c_{1} c_{2}=c_{2}\left(c_{2}^{-1} c_{1} c_{2}\right)$, and thanks to the relations of $G_{\Gamma_{v}}$,

$$
\varepsilon(v)=\widehat{c_{1}} \widehat{c_{2}}=\widehat{c_{2}} \widehat{c_{2}^{-1} c_{1} c_{2}}=\varepsilon\left(v^{\prime}\right) .
$$

The general case for any element of the braid group $\mathcal{B}_{d}$, and for $g^{\prime} \geqslant 0, d \geqslant 2$ follows from a similar calculation for every $\sigma_{j}$ for $1 \leqslant j \leqslant d-1$.

Next, we consider the case where $\varphi$ is a pure mapping class, that is, the case where $\varphi$ does not permute the conjugacy classes associated with the local monodromies. Hence, the first $d$ components of $v$ are conjugate to the first $d$ components of $\varphi \cdot v$; that is, $v_{i} \sim(\varphi \cdot v)_{i}$ for $i=1, \ldots, d$, where $\sim$ denotes conjugation equivalence. We claim that the same is true for the 


\section{Genus STABILIZATION FOR MODULI OF CURVE-SYMMETRIES}

first $d$ components of $\hat{v}$ and $\varphi \cdot \hat{v}$, namely $\hat{v}_{i} \sim(\varphi \cdot \widehat{v})_{i}$ for $i=1, \ldots, d$, where the action of $\widehat{\operatorname{Map}}\left(g^{\prime}, d\right)$ on the set of Hurwitz vectors in $G_{\Gamma_{v}}$ is the one defined in Remark 2.3.

Recall that the action of $\varphi$ on $\Pi_{g^{\prime}, d}$ induces via the isomorphism $\xi$ an action of $\varphi$ on $\mathbb{F}^{\prime}$ and that we have a well-determined extension of this to an automorphism of $\mathbb{F}$ which leaves invariant $E$, which we denote by the same letter $\varphi$. Finally, the action on the set of Hurwitz vectors $\hat{v}$ is obtained by seeing these vectors as morphisms $\mathbb{F} \rightarrow G_{\Gamma}, x_{i} \mapsto \hat{v}_{i}$.

Assume $\varphi\left(\gamma_{i}\right)=\delta_{i}^{-1} \gamma_{i} \delta_{i}$ for $i=1, \ldots, d$. We set $\tilde{\delta}_{i}:=\xi\left(\delta_{i}\right)$ and $\varphi\left(x_{i}\right)=\tilde{\delta}_{i}{ }^{-1} x_{i} \tilde{\delta}_{i}$ for $i=$ $2, \ldots, d$, where $\xi$ is the isomorphism (2.1). In particular, $(\varphi \cdot \hat{v})_{i} \sim \hat{v}_{i}$ for $i=2, \ldots, d$. To define $\varphi\left(x_{1}\right)$, recall that $\xi\left(\gamma_{1}\right)=E^{-1} x_{1}$ in $\mathbb{F}$, so

$$
E^{-1} \varphi\left(x_{1}\right)=\varphi\left(E^{-1} x_{1}\right)=\varphi\left(\xi\left(\gamma_{1}\right)\right)=\xi\left(\varphi\left(\gamma_{1}\right)\right)={\tilde{\delta_{1}}}^{-1} E^{-1} x_{1} \tilde{\delta_{1}} ;
$$

hence $\varphi\left(x_{1}\right)=E{\tilde{\delta_{1}}}^{-1} E^{-1} x_{1} \tilde{\delta_{1}}$. The image of $E$ in $G_{\Gamma_{v}}$ is central, because it belongs to $K_{\Gamma_{v}}$; hence we also have $(\varphi \cdot \hat{v})_{1} \sim \hat{v_{1}}$.

By Lemma 3.3 of [CLP15], we thus have

$$
(\hat{v})_{i} \sim(\varphi \cdot \hat{v})_{i} \Rightarrow(\varphi \cdot \hat{v})_{i}=\alpha\left(\widehat{\left((\varphi \cdot \hat{v})_{i}\right.}\right) .
$$

Now, notice that

$$
\alpha\left((\varphi \cdot \hat{v})_{i}\right)=(\varphi \cdot v)_{i} \quad \text { for } \quad i=1, \ldots, d+2 g^{\prime},
$$

where $\alpha$ is defined in Definition 2.4. This follows by considering Hurwitz vectors as morphisms from the free group $\mathbb{F}$ on $d+2 g^{\prime}$ generators to $G_{\Gamma}$, respectively $G$, and the mapping class group as a group of automorphisms of this free group (cf. Remark 2.3). Therefore, for $i=1, \ldots, d$,

$$
\left.(\varphi \cdot \hat{v})_{i}=\widehat{\alpha\left((\varphi \cdot \hat{v})_{i}\right.}\right)={\widehat{(\varphi \cdot v)_{i}}}_{i}=(\widehat{\varphi \cdot v})_{i} .
$$

Since in the product yielding the evaluation we only take the commutators of the entries $(\varphi \cdot \hat{v})_{i}$ for $i>d$, by Lemma 3.8 of [CLP15] we may freely change the entries $(\varphi \cdot \hat{v})_{i}$ for $i>d$ to $\alpha\left((\varphi \cdot \hat{v})_{i}\right)=(\widehat{\varphi \cdot v})_{i}$, because the statement of Lemma 3.8 is precisely that the value of those commutators remains unchanged. Hence

$$
\operatorname{ev}(\varphi \cdot \hat{v})=\operatorname{ev}(\widehat{\varphi \cdot v})=\varepsilon(\varphi \cdot v) .
$$

Since the evaluation is the image of the element $E$, which is invariant under $\widetilde{\operatorname{Map}}\left(g^{\prime}, d\right)$, we conclude that

$$
\varepsilon(v)=\operatorname{ev}(\hat{v})=\operatorname{ev}(\varphi \cdot \hat{v})=\varepsilon(\varphi \cdot v),
$$

and we have proved our claim.

We also recall that by [CLP15, Lemma 3.5], any automorphism $f \in \operatorname{Aut}(G)$ induces an isomorphism $f_{\Gamma}: K_{\Gamma} \rightarrow K_{f(\Gamma)}$ in a natural way, such that $\varepsilon(f(v))=f_{\Gamma}(\varepsilon(v))$, where $\Gamma=\Gamma_{v}$. Therefore the map

$$
\varepsilon: \operatorname{HS}\left(G ; g^{\prime}, d\right) \rightarrow \coprod_{\Gamma} K_{\Gamma}, \quad v \mapsto \varepsilon(v) \in K_{\Gamma_{v}},
$$

where $\coprod_{\Gamma} K_{\Gamma}$ denotes the disjoint union of the $K_{\Gamma}$, descends to a map

$$
\tilde{\varepsilon}: \operatorname{HS}\left(G ; g^{\prime}, d\right) / \operatorname{Aut}(G) \rightarrow\left(\coprod_{\Gamma} K_{\Gamma}\right) / \operatorname{Aut}(G) .
$$




\section{F. Catanese, M. Lönne and F. Perroni}

Putting these pieces from [CLP15] together, in view of Remark 2.3, gives a well-defined map

$$
\hat{\varepsilon}:\left(\operatorname{HS}\left(G ; g^{\prime}, d\right) / \operatorname{Aut}(G)\right) / \operatorname{Map}\left(g^{\prime}, d\right) \rightarrow\left(\coprod_{\Gamma} K_{\Gamma}\right) / \operatorname{Aut}(G) .
$$

One of the main results of [CLP15] says that when $G=D_{n}$, the map $\hat{\varepsilon}$ is injective ([CLP15, Theorem 5.1]). With the aid of this we have reached a classification of the orbits of Hurwitz generating systems for $D_{n}$ under the action of $\operatorname{Map}\left(g^{\prime}, d\right)$ modulo automorphisms in $\operatorname{Aut}(G)$.

For later use, let us recall the following definition [CLP15, Definition 3.11].

Definition 2.7. Let $\Gamma \subset G$ be a union of non-trivial conjugacy classes of $G$. We define

$$
H_{2, \Gamma}(G)=\operatorname{ker}\left(G_{\Gamma} \rightarrow G \times G_{\Gamma}^{a b}\right),
$$

where $G_{\Gamma} \rightarrow G \times G_{\Gamma}^{a b}$ is the morphism with first component $\alpha$ (Definition 2.4) and second component the natural epimorphism $G_{\Gamma} \rightarrow G_{\Gamma}^{a b}$.

Notice that

$$
H_{2}(G, \mathbb{Z}) \cong \frac{R \cap[F, F]}{[F, R]} \cong \operatorname{ker}\left(F /[F, R] \rightarrow G \times G_{\emptyset}^{a b}\right)
$$

In particular, when $\Gamma=\emptyset$, we have $H_{2, \Gamma}(G) \cong H_{2}(G, \mathbb{Z})$.

By [CLP15, Lemma 3.12], the morphism

$$
R \cap[F, F] \rightarrow R / R_{\Gamma}, \quad r \mapsto r R_{\Gamma}
$$

induces a surjective group homomorphism

$$
H_{2}(G, \mathbb{Z}) \rightarrow H_{2, \Gamma}(G)
$$

\subsection{A topological interpretation of $\varepsilon(v)$}

For a finite group $G$, let $B G$ be the CW-complex defined as follows (see [Whi78, Chapter V, 7] for more details). The 0 -skeleton $B G^{0}$ consists of one point. The 1-skeleton

$$
B G^{1}=\bigvee_{g \in G} S_{g}^{1}
$$

is a wedge of circles indexed by $g \in G$, meeting in $B G^{0}$. Hence its fundamental group has a canonical isomorphism $\pi_{1}\left(B G^{1}\right) \cong F$, given by sending a generator of $\pi_{1}\left(S_{g}^{1}\right)$ to $\hat{g}$. In this way we get an epimorphism $\pi_{1}\left(B G^{1}\right) \rightarrow G$ whose kernel is identified with $R$ by the previous isomorphism. For any $r \in R$, let $h_{r}: S^{1} \rightarrow B G^{1}$ be the continuous map such that the image of a chosen generator of $\pi_{1}\left(S^{1}\right)$ under $\left(h_{r}\right)_{*}$ is $r \in \pi_{1}\left(B G^{1}\right)$. Using $h_{r}$ we attach the 2-cell $E_{r}^{2}$ to $B G^{1}$. This gives the 2 -skeleton $B G^{2}$, with the property that $\pi_{1}\left(B G^{2}\right) \cong G$. The 3 -skeleton $B G^{3}$ is defined by attaching 3-cells to $B G^{2}$ in such a way that $\pi_{2}\left(B G^{3}\right)=0$ and, by induction, the $(n+1)$-skeleton $B G^{n+1}$ is defined similarly in such a way that $\pi_{n}\left(B G^{n+1}\right)=0$ for $n \geqslant 2$. The CW-complex $B G$ is the resulting inductive limit $B G:=\cup\left\{B G^{n} \mid n \geqslant 0\right\}$. By construction $B G$ is an Eilenberg-Mac Lane space of type $(G, 1)$, that is, a $K(G, 1)$-space. Furthermore, there is a principal $G$-bundle $E G \rightarrow B G$ with $E G$ contractible.

The following lemma is a refinement of Hopf's theorem (see [Hop42]) saying that there is an isomorphism

$$
H_{2}(B G, \mathbb{Z}) \rightarrow \frac{R \cap[F, F]}{[R, F]}
$$




\section{Genus STABILIZATION FOR MODULI OF CURVE-SYMMETRIES}

Lemma 2.8. Let $G$ be a finite group, and let $B G$ be the $C W$-complex defined above. There is an isomorphism

$$
R /[F, R] \cong H_{2}\left(B G, B G^{1}\right)
$$

such that the following diagram is commutative:

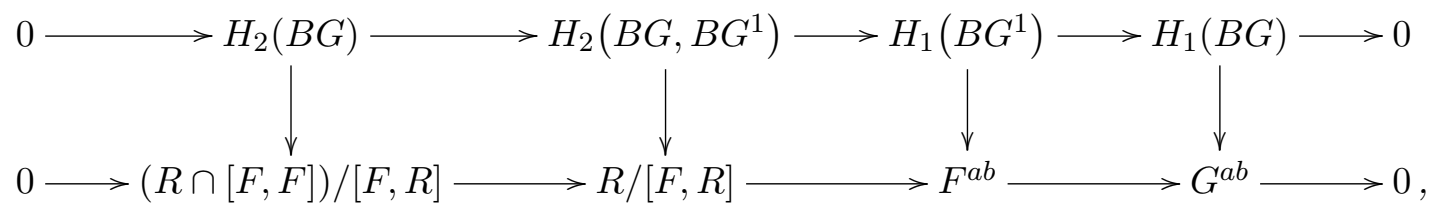

where all the homology groups are with integer coefficients, the horizontal sequences are exact (the upper one being part of the homology sequence of the pair $\left(B G, B G^{1}\right)$ ), and the vertical arrows are isomorphisms (the one on the left is given by Hopf's theorem, the two on the right are the canonical isomorphisms $\left.H_{1} \cong \pi_{1}^{a b}\right)$.

Proof. Recall that the homology of a CW-complex $K=\left\{K^{n}\right\}_{n \in \mathbb{N}}$ can be computed as follows (cf. [Mas91, Chapter IX]). Define

$$
\begin{aligned}
& C_{n}(K):=H_{n}\left(K^{n}, K^{n-1}\right), \\
& \partial_{n}: C_{n}(K) \rightarrow C_{n-1}(K), \quad \partial_{n}=j_{n-1} \circ \partial_{*},
\end{aligned}
$$

where $\partial_{*}: H_{n}\left(K^{n}, K^{n-1}\right) \rightarrow H_{n-1}\left(K^{n-1}\right)$ is the boundary operator of the long exact sequence of the pair $\left(K^{n}, K^{n-1}\right)$ and $j_{n-1}: H_{n-1}\left(K^{n-1}\right) \rightarrow H_{n-1}\left(K^{n-1}, K^{n-2}\right)$ is the homomorphism induced by the inclusion map. Then $\left\{C_{\bullet}(K), \partial_{\bullet}\right\}$ is a complex and $H_{n}(K) \cong H_{n}\left(C_{\bullet}(K)\right)$.

We regard $B G^{1}$ as a 1-dimensional $\mathrm{CW}$-complex, so the above construction gives the complexes $C_{\bullet}(B G)$ and $C_{\bullet}\left(B G^{1}\right)$. Notice that the inclusion $B G^{1} \rightarrow B G$ gives an injective map $C_{\bullet}\left(B G^{1}\right) \rightarrow C_{\bullet}(B G)$ and define

$$
C \bullet\left(B G, B G^{1}\right):=C_{\bullet}(B G) / C \bullet\left(B G^{1}\right) .
$$

Since $C_{n}(K)$ is the free group with basis in 1-1 correspondence with the $n$-cells of $K$, we obtain the diagram

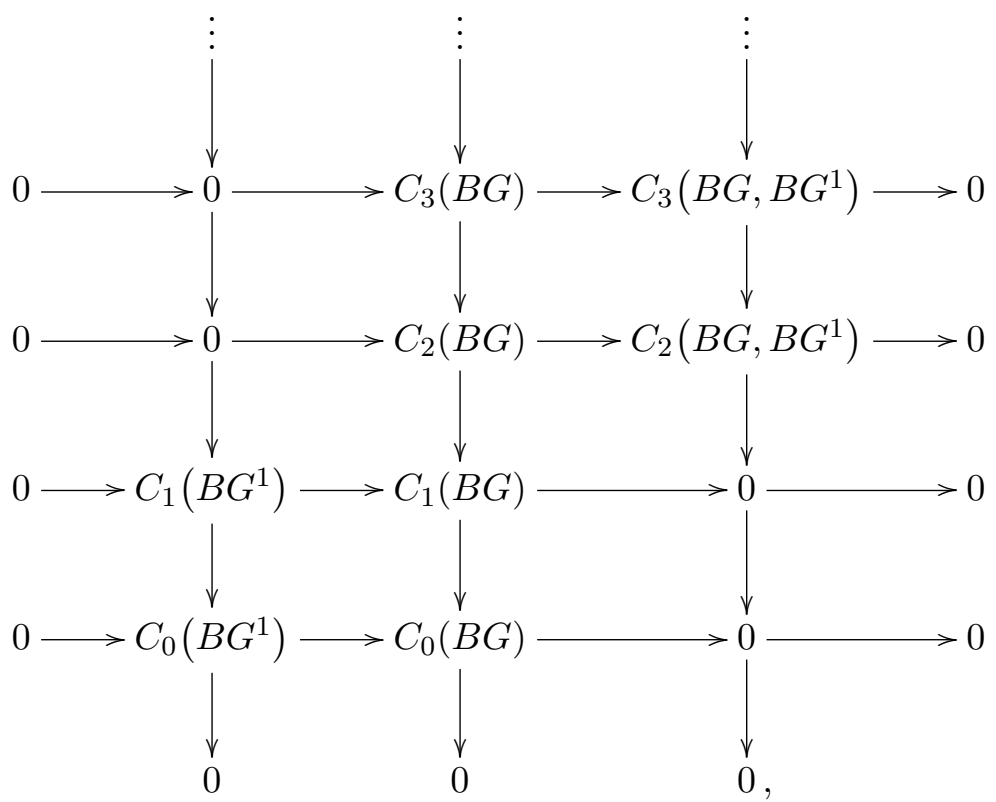




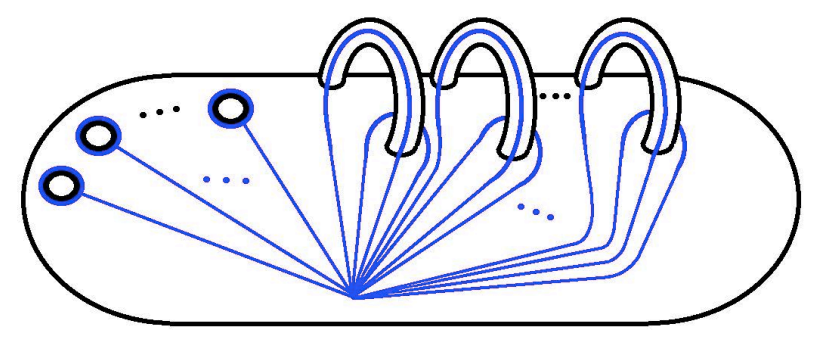

FiguRE 1.

where all rows are exact. Using the isomorphisms $H_{n}(K) \cong H_{n}\left(C_{\bullet}(K)\right)$, the long exact homology sequence of the pair $\left(B G, B G^{1}\right)$, and the long exact homology sequence associated with the previous diagram, we obtain isomorphisms

$$
H_{n}\left(C \bullet\left(B G, B G^{1}\right)\right) \cong H_{n}\left(B G, B G^{1}\right) \text { for all } n .
$$

Consider now the morphism $C_{2}(B G) \rightarrow R /[F, R]$ induced by sending any element of $C_{2}(B G)$ to the homotopy class of its boundary. By (2.4) we have isomorphisms $C_{n}\left(B G, B G^{1}\right) \rightarrow C_{n}(B G)$ for $n \geqslant 2$, and so we obtain a group homomorphism

$$
C_{2}\left(B G, B G^{1}\right) \rightarrow R /[F, R]
$$

By [Hop42, Satz I] it follows that the kernel of $(2.5)$ is $\partial\left(C_{3}\left(B G, B G^{1}\right)\right)=\partial\left(C_{3}(B G)\right)$. So we get the homomorphism

$$
H_{2}\left(B G, B G^{1}\right) \rightarrow R /[F, R]
$$

By construction, the diagram in the statement commutes and (2.6) is an isomorphism by the 5-lemma.

Let now $p: C \rightarrow C^{\prime}$ be a $G$-covering branched at $y_{1}, \ldots, y_{d} \in C^{\prime}$. Fix once and for all a point $y_{0} \in C^{\prime} \backslash\left\{y_{1}, \ldots, y_{d}\right\}$ and a geometric basis $\gamma_{1}, \ldots, \gamma_{d}, \alpha_{1}, \beta_{1}, \ldots, \alpha_{g^{\prime}}, \beta_{g^{\prime}}$ of $\pi_{1}\left(C^{\prime} \backslash\left\{y_{1}, \ldots y_{d}\right\}, y_{0}\right)$. The monodromy of $p$ evaluated at the chosen geometric basis gives the Hurwitz generating system $v \in \operatorname{HS}\left(G ; g^{\prime}, d\right)$, well defined up to conjugation, which in turn determines $p$ (by Riemann's existence theorem). For $\Gamma=\Gamma_{v}$, the $\varepsilon$-invariant of $v$ is an element of

$$
K_{\Gamma}:=R / R_{\Gamma}=(R /[F, R]) /\left\langle\left\langle\hat{a} \hat{b} \hat{c}^{-1} \hat{b}^{-1} \mid a \in \Gamma, a b=b c\right\rangle\right\rangle .
$$

If $p$ is unramified, then, under the identification $(R \cap[F, F]) /[F, R] \cong H_{2}(B G, \mathbb{Z})$, the element $\varepsilon(v)$ of $(R \cap[F, F]) /[F, R] \cong H_{2}(B G, \mathbb{Z})$ coincides with the image $B p_{*}\left[C^{\prime}\right] \in H_{2}(B G, \mathbb{Z})$ of the fundamental class of $C^{\prime}$ under the morphism induced in homology by a classifying map $B p: C^{\prime} \rightarrow$ $B G$ of $p$. We want to extend this topological interpretation of the $\varepsilon$-invariant to the ramified case.

By definition, any loop $\gamma_{i}$ of the geometric basis consists of a path $\tilde{\gamma}_{i}$ from $y_{0}$ to a point $z_{i}$ near $y_{i}$ and of a small loop around $y_{i}$. Let $\Sigma$ be the Riemann surface (with boundary) obtained from $C^{\prime}$ after removing the open discs surrounded by these small loops. Fix once and for all a CW-decomposition of $\Sigma$ as follows. The 0-skeleton $\Sigma^{0}$ consists of the point $y_{0}$ and, for any $i=1, \ldots, d$, the intersection $z_{i}$ of $\tilde{\gamma}_{i}$ and the small circle of $\gamma_{i}$ around $y_{i}$. The 1 -skeleton $\Sigma^{1}$ is given by the geometric basis and the 2 -skeleton $\Sigma^{2}$ consists of one cell (see Figure 1).

The restriction $p_{\Sigma}$ of $p: C \rightarrow C^{\prime}$ to $p^{-1}(\Sigma)$ is an unramified $G$-covering of $\Sigma$ and hence corresponds to a continuous map $B p_{\Sigma}: \Sigma \rightarrow B G$, well defined up to homotopy. Let $B p_{1}: \Sigma \rightarrow B G$ be a cellular approximation of $B p_{\Sigma}$. Since $B p_{1}$ can be regarded as a map of pairs $B p_{1}:(\Sigma, \partial \Sigma) \rightarrow$ 


\section{Genus STABILIZATION FOR MODULi OF CURVE-SyMmetries}

$\left(B G, B G^{1}\right)$, the push-forward of the fundamental (orientation) class $[\Sigma, \partial \Sigma]$ gives an element

$$
B p_{1 *}[\Sigma, \partial \Sigma] \in H_{2}\left(B G, B G^{1}\right)=R /[F, R] .
$$

This element depends on the chosen cellular approximation $B p_{1}$ of $B p_{\Sigma}$, but its image in $K_{\Gamma}$ does not (see Lemma 2.9).

In order to compute $B p_{1_{*}}[\Sigma, \partial \Sigma]$ it is useful to recall the construction of $B p_{1}$. As before, let

$$
v=\left(c_{1}, \ldots, c_{d}, a_{1}, b_{1}, \ldots, a_{g^{\prime}}, b_{g^{\prime}}\right) \in G^{d+2 g^{\prime}}
$$

be a Hurwitz generating system of $p$ with respect to the given geometric basis. Consider the relation

$$
r:=\widehat{c_{1}} \cdots \widehat{c_{d}} \cdot \prod_{j=1}^{g^{\prime}}\left[\widehat{a_{j}}, \widehat{b_{j}}\right] \in \pi_{1}\left(B G^{1}\right)
$$

and a continuous map $h_{r}: \Sigma^{1} \rightarrow B G^{1}$ representing $r$. By the construction of $B G$ there is a 2cell $E_{r}$ attached along $h_{r}$. Choose a homeomorphism $E_{r} \cong \Sigma^{2}$. So we get a continuous map $B p_{1}: \Sigma \rightarrow B G$. To prove that $B p_{1}$ is homotopic to $B p$, we need to prove that the pull-back $B p_{1}^{*}(E G \rightarrow B G)$ under $B p_{1}$ of $E G \rightarrow B G$ is $p_{\Sigma}: p^{-1}(\Sigma) \rightarrow \Sigma$. This follows from the fact that $E G \rightarrow B G$ is the universal cover and from the fact that the monodromy vector of $B p_{1}^{*}(E G \rightarrow$ $B G)$ with respect to the given geometric basis coincides with $v$ up to conjugation. Moreover, by the proof of Lemma 2.8, it follows that under the isomorphism $H_{2}\left(B G, B G^{1}\right) \cong R /[F, R]$,

$$
B p_{1 *}[\Sigma, \partial \Sigma]=\prod_{1}^{d} \widehat{c_{i}} \cdot \prod_{1}^{g^{\prime}}\left[\widehat{a_{j}}, \widehat{b_{j}}\right] \in R /[F, R] .
$$

Lemma 2.9. Let $\Gamma=\Gamma_{v} \subset G$, where $v$ is the Hurwitz generating system of $p$ with respect to the given geometric basis. Then the image of $B p_{1_{*}}[\Sigma, \partial \Sigma]$ in $K_{\Gamma}$ does not depend on the cellular approximation $B p_{1}$ of $B p$. Denote this element by $B p_{*}[\Sigma, \partial \Sigma] \in K_{\Gamma}$. Then, by (2.7) we have

$$
B p_{*}[\Sigma, \partial \Sigma]=\varepsilon(v) \in K_{\Gamma} .
$$

Proof. Let $B p_{2}:(\Sigma, \partial \Sigma) \rightarrow\left(B G, B G^{1}\right)$ be another cellular approximation of $B p_{\Sigma}$. Then there exists a homotopy $F: I \times \Sigma \rightarrow B G$ such that $F(0, x)=B p_{1}(x)$ and $F(1, x)=B p_{2}(x)$ for all $x \in \Sigma$, where $I=[0,1]$. Without loss of generality, we may assume that $F$ is cellular with respect to the standard CW-decomposition of $I \times \Sigma[$ Whi78, (4.7)]:

$$
(I \times \Sigma)^{n}=\bigcup_{i=0}^{n} I^{i} \times \Sigma^{n-i},
$$

where $I$ is seen as a 1-dimensional CW-complex with $I^{0}=\{0\} \cup\{1\}$.

Consider now the chain homotopy

$$
\varphi_{n}: C_{n}(B G) \rightarrow C_{n+1}(B G)
$$

between the chain maps $B p_{1 \#}$ and $B p_{2 \#}$ associated with $F$. Then we have (cf. [Mas91, (7.4.1)])

$$
B p_{1 \#}-B p_{2 \#}=\partial_{n+1} \circ \varphi_{n}+\varphi_{n-1} \circ \partial_{n} \text {. }
$$

From this it follows that

$$
B p_{1 *}[\Sigma, \partial \Sigma]-B p_{2 *}[\Sigma, \partial \Sigma]=\left[\varphi_{1}(\partial \Sigma)\right] \in H_{2}\left(B G, B G^{1}\right) .
$$

Notice that

$$
\left[\varphi_{1}(\partial \Sigma)\right]=\left(F_{\mid I \times \partial \Sigma}\right)_{*}[I \times \partial \Sigma, \partial(I \times \partial \Sigma)] \in H_{2}\left(B G, B G^{1}\right) .
$$




\section{F. Catanese, M. Lönne and F. Perroni}

The claim now follows from the fact that $I \times \partial \Sigma$ is the union of cylinders $I \times S^{1}$ and

$$
\left(F_{\mid I \times \partial \Sigma}\right)_{*}\left[I \times S^{1}, \partial\left(I \times S^{1}\right)\right]=\hat{a} \hat{b} \hat{c}^{-1} \hat{b}^{-1},
$$

where $\hat{a}$ (respectively, $\hat{c}$ ) is the image of the fundamental class of $\{0\} \times S^{1}$ (respectively, $\{1\} \times S^{1}$ ) under $F$ and $\hat{b}$ is the image of $I \times\left\{z_{i}\right\}$ under $F$ for some $z_{i} \in \Sigma^{0}$. Notice that, since the image $\left(F_{\mid I \times \partial \Sigma}\right)_{*}\left[I \times S^{1}, \partial\left(I \times S^{1}\right)\right]$ is the class of a 2 -cell, $\hat{a} \hat{b} \hat{c}^{-1} \hat{b}^{-1}$ must be a relation for $G$.

\section{The main theorem}

In this section we prove our main result. Roughly speaking, it says that for $g^{\prime}$ sufficiently large, the map given by the $\hat{\varepsilon}$-invariant (2.2) is injective and its image is independent of $g^{\prime}$ and coincides with the classes of admissible $\nu$-types. We begin with the following definition.

Definition 3.1. Let $v \in \operatorname{HS}\left(G ; g^{\prime}, d\right)$, and let $\nu(v) \in \bigoplus_{\mathcal{C}} \mathbb{Z}\langle\mathcal{C}\rangle(\mathcal{C}$ runs over the set of non-trivial conjugacy classes of $G$ ) be the vector whose $\mathcal{C}$-component is the number of $v_{j}$ for $j \leqslant d$ which belong to $\mathcal{C}$. The map

$$
\nu: \operatorname{HS}\left(G ; g^{\prime}, d\right) \rightarrow \bigoplus_{\mathcal{C}} \mathbb{Z}\langle\mathcal{C}\rangle
$$

obtained in this way induces a map

$$
\tilde{\nu}: \operatorname{HS}\left(G ; g^{\prime}, d\right) / \operatorname{Aut}(G) \rightarrow\left(\bigoplus_{\mathcal{C}} \mathbb{Z}\langle\mathcal{C}\rangle\right) / \operatorname{Aut}(G)
$$

which is $\operatorname{Map}\left(g^{\prime}, d\right)$-invariant; therefore we get a map

$$
\hat{\nu}:\left(\operatorname{HS}\left(G ; g^{\prime}, d\right) / \operatorname{Aut}(G)\right) / \operatorname{Map}\left(g^{\prime}, d\right) \rightarrow\left(\bigoplus_{\mathcal{C}} \mathbb{Z}\langle\mathcal{C}\rangle\right) / \operatorname{Aut}(G) .
$$

For any $v \in \operatorname{HS}\left(G ; g^{\prime}, d\right)$, we call $\hat{\nu}(v)$ the (unmarked) $\nu$-type of $v$ (the marked version $\nu(v)$ is called the shape in [FV91]).

Remark 3.2. Let $v \in \operatorname{HS}\left(G ; g^{\prime}, d\right)$, and let $\Gamma_{v} \subset G$ be the union of the conjugacy classes of the $v_{j}$ for $j \leqslant d$. The abelianization $G_{\Gamma_{v}}^{a b}$ of $G_{\Gamma_{v}}$ can be described as follows:

$$
G_{\Gamma_{v}}^{a b} \cong \bigoplus_{\mathcal{C} \subset \Gamma_{v}} \mathbb{Z}\langle\mathcal{C}\rangle \bigoplus_{g \in G \backslash \Gamma_{v}} \mathbb{Z}\langle g\rangle
$$

where $\mathcal{C}$ denotes a conjugacy class of $G$. Moreover, $\nu(v)$ coincides with the vector whose $\mathcal{C}$ components are the corresponding components of the image in $G_{\Gamma_{v}}^{a b}$ of $\varepsilon(v) \in G_{\Gamma_{v}}$ under the natural homomorphism $G_{\Gamma_{v}} \rightarrow G_{\Gamma_{v}}^{a b}$. It follows that $\nu$ in (3.1) factors as

$$
\nu=A \circ \varepsilon,
$$

where

$$
A: \coprod_{\Gamma} K_{\Gamma} \rightarrow \bigoplus_{\mathcal{C}} \mathbb{Z}\langle\mathcal{C}\rangle
$$

is induced from the abelianization $G_{\Gamma} \rightarrow G_{\Gamma}^{a b}$. To take into account the automorphisms of $G$ one similarly defines $\widehat{A}$ in such a way that $\widehat{\nu}=\widehat{A} \circ \widehat{\varepsilon}$.

Definition 3.3. An element

$$
\left(n_{\mathcal{C}}\right)_{\mathcal{C}} \in \bigoplus_{\mathcal{C}} \mathbb{Z}\langle\mathcal{C}\rangle
$$




\section{Genus STABILIZATION FOR MODULI OF CURVE-SYMMETRIES}

is admissible if $n_{\mathcal{C}} \geqslant 0$ for all $\mathcal{C}$ and the following equality holds in $G^{a b}$ for its natural $\mathbb{Z}$-module structure:

$$
\sum_{\mathcal{C}} n_{\mathcal{C}} \cdot[\mathcal{C}]=0
$$

where $[\mathcal{C}]$ denotes the class of any element of $\mathcal{C}$ in the abelianization of $G$.

Accordingly, we say that $\widehat{\left(n_{\mathcal{C}}\right)_{\mathcal{C}}} \in\left(\bigoplus_{\mathcal{C}} \mathbb{Z}\langle\mathcal{C}\rangle\right) / \operatorname{Aut}(G)$ is admissible if it is the class of an admissible element.

The main result of the paper is then the following.

Theorem 3.4. Let $G$ be a finite group. For any $d \in \mathbb{N}$, there is an integer $s=s(d)$ such that

$$
\hat{\varepsilon}: \quad\left(\operatorname{HS}\left(G ; g^{\prime}, d\right) / \operatorname{Aut}(G)\right) / \operatorname{Map}\left(g^{\prime}, d\right) \rightarrow\left(\coprod_{\Gamma} K_{\Gamma}\right) / \operatorname{Aut}(G)
$$

is injective for all $g^{\prime}>s$. Moreover, for all $g^{\prime}>s$, the image of $\hat{\varepsilon}$ is independent of $g^{\prime}$ and coincides with the pre-image under $\widehat{A}$ of the admissible $\widehat{\nu}$-types $\widehat{\left(n_{\mathcal{C}}\right)_{\mathcal{C}}} \in\left(\bigoplus_{\mathcal{C}} \mathbb{Z}\langle\mathcal{C}\rangle\right) / \operatorname{Aut}(G)$ such that $\sum_{\mathcal{C}} n_{\mathcal{C}}=d$.

The main tool to understand the set $\left(\operatorname{HS}\left(G ; g^{\prime}, d\right) / \operatorname{Aut}(G)\right) / \operatorname{Map}\left(g^{\prime}, d\right)$ is provided by the so-called stabilization, which we are going to review once more. For any Hurwitz generating system

$$
v=\left(c_{1}, \ldots, c_{d}, a_{1}, b_{1}, \ldots, a_{g^{\prime}}, b_{g^{\prime}}\right) \in \operatorname{HS}\left(G ; g^{\prime}, d\right),
$$

define the $\left(h\right.$-) stabilization $v^{h}$ of $v$ inductively by

$$
v^{0}=v, \quad v^{1}=\left(c_{1}, \ldots, c_{d}, a_{1}, b_{1}, \ldots, a_{g^{\prime}}, b_{g^{\prime}}, 1,1\right), \quad v^{h}=\left(v^{h-1}\right)^{1} \quad \text { for all } h \in \mathbb{N} .
$$

Topologically, if $v$ corresponds to the monodromy $\mu: \pi_{1}\left(C^{\prime}\right) \rightarrow G$, then $v^{h}$ corresponds to the monodromy $\mu^{h}: \pi_{1}\left(C^{\prime} \# C^{\prime \prime}\right) \rightarrow G$ obtained by extending $\mu$ by 1 on the elements of $\pi_{1}\left(C^{\prime \prime}\right)$, where $C^{\prime \prime}$ is an oriented surface of genus $h$ and $C^{\prime} \# C^{\prime \prime}$ is the connected sum of $C^{\prime}$ and $C^{\prime \prime}$.

It is easy to see that stabilization satisfies the following properties: the $\varepsilon$-invariant does not change under stabilization,

$$
\varepsilon(v)=\varepsilon\left(v^{h}\right) \text { for all } v, h,
$$

and it respects the equivalence relation given by the actions of $\operatorname{Aut}(G)$ and $\operatorname{Map}\left(g^{\prime}, d\right)$. Therefore we have maps

$$
\begin{aligned}
\left(\operatorname{HS}\left(G ; g^{\prime}, d\right) / \operatorname{Aut}(G)\right) / \operatorname{Map}\left(g^{\prime}, d\right) & \rightarrow\left(\operatorname{HS}\left(G ; g^{\prime}+h, d\right) / \operatorname{Aut}(G)\right) / \operatorname{Map}\left(g^{\prime}+h, d\right), \quad \forall g^{\prime}, h, d, \\
{[v] } & \mapsto\left[v^{h}\right] .
\end{aligned}
$$

Moreover, the $\varepsilon$-invariant is stably a fine invariant. This is the content of the following theorem that extends to the ramified case the analogous result of Livingston [Liv85] for non-ramified group actions (see also [DT06, Zim87]).

Theorem 3.5. Let $G$ be a finite group, and let $v, w \in \operatorname{HS}\left(G ; g^{\prime}, d\right)$ such that $\nu(v)=\nu(w)$; in particular, $\Gamma_{v}=\Gamma_{w}=\Gamma$. If $\varepsilon(v)=\varepsilon(w) \in K_{\Gamma}$, then there exists an $h \in \mathbb{N}$ such that the classes of $v^{h}$ and $w^{h}$ in $\left(\operatorname{HS}\left(G ; g^{\prime}+h, d\right) / \operatorname{Aut}(G)\right) / \operatorname{Map}\left(g^{\prime}+h, d\right)$ coincide.

We postpone the proof of this theorem to the next section. Here we use it to give a proof of Theorem 3.4. 


\section{F. Catanese, M. Lönne and F. Perroni}

Proof of Theorem 3.4. First of all, for $g^{\prime} \geqslant|G|$, any $v \in \operatorname{HS}\left(G ; g^{\prime}+1, d\right)$ is a stabilization; that is, the map

$$
\begin{aligned}
\varsigma_{g^{\prime}}: \operatorname{HS}\left(G ; g^{\prime}, d\right) / \widetilde{\operatorname{Map}}\left(g^{\prime}, d\right) & \rightarrow \operatorname{HS}\left(G ; g^{\prime}+1, d\right) / \widetilde{\operatorname{Map}}\left(g^{\prime}+1, d\right) \\
\text { induced by } \quad v & \mapsto v^{1}
\end{aligned}
$$

is surjective. This follows from the proof of [DT06, Proposition 6.16], where the result is stated for free group actions, but the same proof works also for non-free actions.

As a consequence, (3.3) is surjective for $g^{\prime} \geqslant|G|$.

Since the sets $\left(\operatorname{HS}\left(G ; g^{\prime}, d\right) / \operatorname{Aut}(G)\right) / \operatorname{Map}\left(g^{\prime}, d\right)$ are finite, there exists an integer ${ }^{1} s=s(d)$ such that (3.3) is bijective for any $g^{\prime}>s$ and $h \geqslant 1$.

Let now $g^{\prime}>s$ and let $v, w \in \operatorname{HS}\left(G ; g^{\prime}, d\right)$ with $\varepsilon(v)=\varepsilon(w)$. From Theorem 3.5 there exists an $h$ with $\left[v^{h}\right]=\left[w^{h}\right] \in\left(\operatorname{HS}\left(G ; g^{\prime}+h, d\right) / \operatorname{Aut}(G)\right) / \operatorname{Map}\left(g^{\prime}+h, d\right)$. Since stabilization is bijective in this range, we get $[v]=[w] \in\left(\operatorname{HS}\left(G ; g^{\prime}, d\right) / \operatorname{Aut}(G)\right) / \operatorname{Map}\left(g^{\prime}, d\right)$. Hence $\widehat{\varepsilon}$ is injective for $g^{\prime}>s$.

The fact that $\operatorname{Im}(\widehat{\varepsilon})$ does not depend on $g^{\prime}$ now follows from (3.2).

We now prove that $\operatorname{Im}(\widehat{\varepsilon})$ is the pre-image under $\widehat{A}$ of the admissible $\widehat{\nu}$-types

$$
\widehat{\left(n_{\mathcal{C}}\right)_{\mathcal{C}}} \in\left(\bigoplus_{\mathcal{C}} \mathbb{Z}\langle\mathcal{C}\rangle\right) / \operatorname{Aut}(G)
$$

such that $\sum_{\mathcal{C}} n_{\mathcal{C}}=d$ for any $g^{\prime}>s$. First, notice that for any representative $\left(n_{\mathcal{C}}\right)_{\mathcal{C}} \in \bigoplus_{\mathcal{C}} \mathbb{Z}\langle\mathcal{C}\rangle$ of such an admissible $\hat{\nu}$-type, there exists a $v \in \operatorname{HS}(G ; s+1, d)$ with $\nu(v)=\left(n_{\mathcal{C}}\right)_{\mathcal{C}}$.

Indeed, there exists a $\left(c_{1}, \ldots, c_{d}\right)$ with $\nu\left(c_{1}, \ldots, c_{d}\right)=\left(n_{\mathcal{C}}\right)_{\mathcal{C}}$ because $n_{\mathcal{C}} \geqslant 0$ for all $\mathcal{C}$. And for any such $\left(c_{1}, \ldots, c_{d}\right)$, the condition to be admissible implies that $c_{1} \cdots c_{d} \in[G, G]$, hence this is a product of commutators:

$$
c_{1} \cdots c_{d}=\left(\prod_{j=1}^{r}\left[a_{j}, b_{j}\right]\right)^{-1}
$$

for some $r \in \mathbb{N}$. If $c_{1}, \ldots, c_{d}, a_{1}, b_{1}, \ldots, a_{r}, b_{r}$ do not generate $G$, we add pairs of the form $(g, 1)$, so we obtain a Hurwitz generating system $w \in \operatorname{HS}(G ; r, d)$ with $\nu(w)=\left(n_{\mathcal{C}}\right)_{\mathcal{C}}$. Moreover, we may assume $r>s$. Since $\varsigma_{g^{\prime}}$ is surjective for $g^{\prime}>s$, we get a Hurwitz system $v \in \operatorname{HS}(G ; s+1, d)$ whose stabilization is $\widetilde{\operatorname{Map}}\left(g^{\prime}, d\right)$-equivalent to $w$ and the claim follows.

Finally, we prove that for any $\xi \in K_{\Gamma}$ for $\Gamma=\Gamma_{v}$ with $A(\xi)=\left(n_{\mathcal{C}}\right)_{\mathcal{C}}$, there exists a $w \in$ $\operatorname{HS}(G ; s+1, d)$ with $\varepsilon(w)=\xi$. Since $A(\xi)=A(\varepsilon(v))=\left(n_{\mathcal{C}}\right)_{\mathcal{C}}$, we have $\varepsilon(v)^{-1} \cdot \xi \in H_{2, \Gamma}(G)$. By (2.3) there exists an $\eta \in H_{2}(G, \mathbb{Z})$ which maps to $\varepsilon(v)^{-1} \cdot \xi$. Since bordism is the same as homology in dimension 2 (cf. also [DT06, Theorem 6.20]), there is a Hurwitz system $v^{\prime} \in$ $\operatorname{HS}(G ; h, 0)$ such that $\varepsilon\left(v^{\prime}\right)=\eta \in H_{2}(G, \mathbb{Z})$. Let $v^{\prime \prime} \in \operatorname{HS}(G ; s+1+h, d)$ be the system whose first $d+2(s+1)$ components coincide with those of $v$ and whose last $2 h$ components are those of $v^{\prime}$; then we have $\varepsilon\left(v^{\prime \prime}\right)=\xi$. By (3.2) the system $w \in \operatorname{HS}(G ; s+1, d)$ that maps to $v^{\prime \prime}$ under $\varsigma_{s+h} \circ \cdots \circ \varsigma_{s+1}$ satisfies $\varepsilon(w)=\xi$. This concludes the proof of the theorem.

\footnotetext{
${ }^{1}$ To prove that $s$ can be explicitly given it would suffice to show that once $\varsigma_{g^{\prime}}$ is bijective, $\varsigma_{g^{\prime \prime}}$ is also bijective for $g^{\prime \prime}>g^{\prime}$.
} 


\section{Genus STABILIZATION FOR MODULI OF CURVE-SYMMETRIES}

\section{Proof of Theorem 3.5}

Let $v, w \in \operatorname{HS}\left(G, g^{\prime}, d\right)$ be two Hurwitz generating systems with $\nu(v)=\nu(w)$ and $\varepsilon(v)=\varepsilon(w)$. If

$$
\text { and } \quad \begin{aligned}
v & =\left(v_{1}, \ldots, v_{d} ; v_{d+1}, v_{d+2}, \ldots, v_{d+2 g^{\prime}-1}, v_{d+2 g^{\prime}}\right) \\
w & =\left(w_{1}, \ldots, w_{d} ; w_{d+1}, w_{d+2}, \ldots, w_{d+2 g^{\prime}-1}, w_{d+2 g^{\prime}}\right),
\end{aligned}
$$

then, without loss of generality (using braid group moves on the first $d$ components), we may assume that $v_{i}$ is conjugate to $w_{i}$ for $i=1, \ldots, d$, and that the following equality holds:

$$
\prod_{i=1}^{d} \widehat{w_{i}} \prod_{j=1}^{g^{\prime}}\left[\widehat{w_{d+2 j-1}}, \widehat{w_{d+2 j}}\right] \equiv \prod_{i=1}^{d} \widehat{v_{i}} \prod_{j=1}^{g^{\prime}}\left[\widehat{v_{d+2 j-1}}, \widehat{v_{d+2 j}}\right] \quad\left(\bmod R_{\Gamma}\right) .
$$

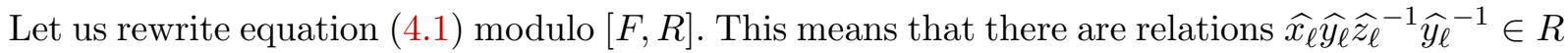
for $\ell=1, \ldots, N$, with $x_{\ell}, z_{\ell} \in \Gamma$, such that

$$
\prod_{i=1}^{d} \widehat{w_{i}} \prod_{j=1}^{g^{\prime}}\left[\widehat{w_{d+2 j-1}}, \widehat{w_{d+2 j}}\right] \equiv \prod_{i=1}^{d} \widehat{v_{i}} \prod_{j=1}^{g^{\prime}}\left[\widehat{v_{d+2 j-1}}, \widehat{v_{d+2} j}\right] \prod_{\ell=1}^{N}\left(\widehat{x_{\ell}} \widehat{y_{\ell}}{\widehat{z_{\ell}}}^{-1}{\widehat{y_{\ell}}}^{-1}\right)^{ \pm 1} \quad(\bmod [F, R]) \text {. }
$$

Proposition 4.1. Let $v, w \in \operatorname{HS}\left(G, g^{\prime}, d\right)$ be two Hurwitz generating systems. Assume that the following hypotheses are satisfied:

(i) $v_{i}=w_{i}$ for $i=1, \ldots, d$;

(ii) $\operatorname{ev}(\hat{v}) \equiv \operatorname{ev}(\hat{w}) \quad(\bmod [F, R])($ that is, $N=0$ in $(4.2))$;

(iii) $G=\left\langle v_{d+1}, \ldots, v_{d+2 g^{\prime}}\right\rangle=\left\langle w_{d+1}, \ldots, w_{d+2 g^{\prime}}\right\rangle$.

Then $v$ and $w$ are stably equivalent.

Proof. We have

$$
\operatorname{ev}(\hat{w})=\prod_{i=1}^{d} \widehat{v_{i}} \prod_{j=1}^{g^{\prime}}\left[\widehat{w_{d+2 j}-1}, \widehat{w_{d+2 j}}\right] \equiv \operatorname{ev}(\hat{v})=\prod_{i=1}^{d} \widehat{v_{i}} \prod_{j=1}^{g^{\prime}}\left[\widehat{v_{d+2 j-1}}, \widehat{v_{d+2 j}}\right] \quad(\bmod [F, R]),
$$

hence

$$
\prod_{j=1}^{g^{\prime}}\left[\widehat{w_{d+2 j-1}}, \widehat{w_{d+2 j}}\right] \equiv \prod_{j=1}^{g^{\prime}}\left[\widehat{v_{d+2 j-1}}, \widehat{v_{d+2 j}}\right] \quad(\bmod [F, R])
$$

Recall Miller's result in [Mil52]:

$$
H_{2}(G, \mathbb{Z})=Z(G) / B(G),
$$

where $Z(G)$ and $B(G)$ are defined as follows. Miller considers the free group $\langle G, G\rangle$ on all pairs $\langle x, y\rangle$ with $x, y \in G$ (here we follow the notation in [Mil52] and [Zim87, §1, p. 249]), which has a natural homomorphism

$$
e:\langle G, G\rangle \longrightarrow[G, G], \quad\langle x, y\rangle \mapsto[x, y] .
$$

Then $Z(G)$ is defined as $\operatorname{ker}(e)$.

By the same arguments as those following [Mil52, Theorem 2, p. 593], there is another natural homomorphism

$$
\langle G, G\rangle \rightarrow F /[F, R], \quad\langle x, y\rangle \mapsto[\hat{x}, \hat{y}],
$$

which has kernel $B(G)$, where $B(G)$ is normally generated by the relations $R_{i}$ appearing in formulas (1.1)-(1.4) of [Zim87]. 


\section{F. Catanese, M. Lönne and F. Perroni}

Let us now consider the elements

$$
\bar{\Omega}(w):=\prod_{j=1}^{g^{\prime}}\left\langle w_{d+2 j-1}, w_{d+2 j}\right\rangle, \quad \bar{\Omega}(v):=\prod_{j=1}^{g^{\prime}}\left\langle v_{d+2 j-1}, v_{d+2 j}\right\rangle .
$$

Then (4.3) can be interpreted as saying

$$
\bar{\Omega}(w) \equiv \bar{\Omega}(v) \quad(\bmod B(G)) .
$$

To show from this property that $v$ and $w$ are stably equivalent, one could refer to [Liv85, Zim87] in the special case where both sides are in $Z(G)=\operatorname{ker}(\langle G, G\rangle \rightarrow[G, G])$. But, since we need to establish a more general claim, we elaborate on the proof given in [Zim87].

By hypothesis (4.4) we have

$$
\bar{\Omega}(v)=\bar{\Omega}(w) X_{1} R_{1} X_{1}^{-1} X_{2} R_{2} X_{2}^{-1} \cdots \in\langle G, G\rangle,
$$

where $X_{i} \in\langle G, G\rangle$ and the $R_{i}$ are the relations (1.1)-(1.4) of [Zim87] or their inverses (notice that in formula (1.4) of [Zim87], $\langle x, y\rangle^{x}$ should read $\left.\langle y, z\rangle^{x}\right)$. Let us write the right-hand side of $(4.5)$ as

$$
\prod_{j=1}^{M}\left\langle u_{j}, u_{j}^{\prime}\right\rangle^{\epsilon_{j}}
$$

where $\epsilon_{j}=1$ and $\left(u_{j}, u_{j}^{\prime}\right)=\left(w_{d+2 j-1}, w_{d+2 j}\right)$ if $j=1, \ldots, g^{\prime}$, while for $j>g^{\prime}$ we have

$$
\begin{aligned}
\left\langle u_{g^{\prime}+1}, u_{g^{\prime}+1}^{\prime}\right\rangle^{\epsilon_{g^{\prime}+1}} \cdots\left\langle u_{k_{1}}, u_{k_{1}}^{\prime}\right\rangle^{\epsilon_{k_{1}}}=X_{1}, \\
\left\langle u_{k_{1}+1}, u_{k_{1}+1}^{\prime}\right\rangle^{\epsilon_{k_{1}+1}} \cdots\left\langle u_{k_{2}}, u_{k_{2}}^{\prime}\right\rangle^{\epsilon_{k_{2}}}=R_{1}, \\
\left\langle u_{k_{2}+1}, u_{k_{2}+1}^{\prime}\right\rangle^{\epsilon_{k_{2}+1}} \cdots\left\langle u_{k_{3}}, u_{k_{3}}^{\prime}\right\rangle^{\epsilon_{k_{3}}}=X_{1}^{-1},
\end{aligned}
$$

and so on. The $\epsilon_{j}$ are all +1 or -1 . We obviously have, for instance, $\left\langle u_{k_{3}}, u_{k_{3}}^{\prime}\right\rangle=\left\langle u_{g^{\prime}+1}, u_{g^{\prime}+1}^{\prime}\right\rangle$ and $\epsilon_{k_{3}} \epsilon_{g^{\prime}+1}=-1$, and similarly for the other factors.

Let us consider the étale Hurwitz vector

$$
w^{\prime}=\left(z_{1}, z_{1}^{\prime}, z_{2}, z_{2}^{\prime}, \ldots, z_{M}, z_{M}^{\prime}\right),
$$

where $\left(z_{j}, z_{j}^{\prime}\right)=\left(u_{j}, u_{j}^{\prime}\right)$ if $\epsilon_{j}=1$, while $\left(z_{j}, z_{j}^{\prime}\right)=\left(u_{j}^{\prime}, u_{j}\right)$ if $\epsilon_{j}=-1$. Notice that, by hypothesis (iii), the components of $w^{\prime}$ generate $G$. Define $v_{\text {et }}:=\left(v_{d+1}, v_{d+2}, \ldots, v_{2 g^{\prime}-1}, v_{2 g^{\prime}}\right)$ and $w_{\text {et }}:=\left(w_{d+1}, w_{d+2}, \ldots, w_{2 g^{\prime}-1}, w_{2 g^{\prime}}\right)$. We now proceed as follows: we first prove that $v_{\text {et }}$ is stably equivalent to $w^{\prime}$, and then that $w^{\prime}$ is stably equivalent to $w_{\text {et }}$.

Step 1: $v_{\text {et }}$ is stably equivalent to $w^{\prime}$. The element $\bar{\Omega}(v)$ is a positive word. Hence, after cancelling pairs of consecutive letters of type $\left\langle u, u^{\prime}\right\rangle\left\langle u, u^{\prime}\right\rangle^{-1}$ or $\left\langle u, u^{\prime}\right\rangle^{-1}\left\langle u, u^{\prime}\right\rangle$ in (4.6), we end with the positive word $\bar{\Omega}(v)$ again.

Notice that pairs of consecutive letters like $\left\langle u, u^{\prime}\right\rangle\left\langle u, u^{\prime}\right\rangle^{-1}$ or $\left\langle u, u^{\prime}\right\rangle^{-1}\left\langle u, u^{\prime}\right\rangle$ in (4.6) correspond to 4 -tuples of consecutive coordinates of $w^{\prime}$ like $\left(u, u^{\prime}, u^{\prime}, u\right)$ or $\left(u^{\prime}, u, u, u^{\prime}\right)$, by the construction of $w^{\prime}$. Now, we can use Zimmermann's moves to transform these 4 -tuples to $(1,1,1,1)$. Indeed, a direct computation shows that any automorphism $\varphi$ of the form (2.1)-(2.5) in [Zim87] is induced by an automorphism $\tilde{\varphi}$ of $\Pi_{g^{\prime}, d}$ such that $\tilde{\varphi}\left(\gamma_{i}\right)=\gamma_{i}$ for $i=1, \ldots, d$. Hence by the extended Dehn-Nielsen theorem (see, for example, [ACG11, Chapter 15, §3] and the references therein), $\tilde{\varphi}$ is induced by an element of $\widetilde{\operatorname{Map}}\left(g^{\prime}, d\right)$.

Since $v_{\text {et }}$ is a Hurwitz vector for which (4.5) holds and whose components generate $G$ (by hypothesis (iii)), we obtain again a Hurwitz vector whose components generate $G$ each time we apply the previous transformation to $w^{\prime}$. So the procedure can be repeated. 


\section{Genus STABILIZATION FOR MODUli OF CURVE-SYMMETRIES}

Step 2: $w^{\prime}$ is stably equivalent to $w_{\text {et. }}$. We prove this statement in the case where there is only one relation $R_{i}$ on the right-hand side of (4.5), that is, when

$$
\bar{\Omega}(v)=\bar{\Omega}(w) X R X^{-1} \in\langle G, G\rangle,
$$

where $X \in\langle G, G\rangle$ and $R$ is one of the relations (1.1)-(1.4) of [Zim87] or their inverses. The general case follows from this by induction on the number of relations $R_{i}$.

So with $w_{\text {et }}=\left(u_{1}, u_{1}^{\prime}, \ldots, u_{g^{\prime}}, u_{g^{\prime}}^{\prime}\right)$ as before we can write

$$
\begin{aligned}
w^{\prime}= & \left(u_{1}, u_{1}^{\prime}, \ldots, u_{g^{\prime}}, u_{g^{\prime}}^{\prime}, z_{g^{\prime}+1}, z_{g^{\prime}+1}^{\prime}, \ldots, z_{k_{1}}, z_{k_{1}}^{\prime},\right. \\
& \left.z_{k_{1}+1}, z_{k_{1}+1}^{\prime}, \ldots, z_{k_{2}}, z_{k_{2}}^{\prime}, z_{k_{1}}^{\prime}, z_{k_{1}}, \ldots, z_{g^{\prime}+1}^{\prime}, z_{g^{\prime}+1}\right),
\end{aligned}
$$

where the coordinates $\left(z_{g^{\prime}+1}, z_{g^{\prime}+1}^{\prime}, \ldots, z_{k_{1}}, z_{k_{1}}^{\prime}\right)$ correspond to $X \in\langle G, G\rangle$.

The part $\left(z_{k_{1}+1}, z_{k_{1}+1}^{\prime}, \ldots, z_{k_{2}}, z_{k_{2}}^{\prime}\right)$ corresponds to $R$, hence it has one of the following forms (we follow the convention that the relations of Zimmermann are rewritten as relators by multiplying the right-hand side by the inverse of the left-hand side):

(i) $(x, x)$;

(ii) $(x, y, y, x)$;

(iii) $\left(z, x y, y^{x}, z^{x}, x, z\right)$ or $\left(z, x, z^{x}, y^{x}, x y, z\right)$;

(iv) $\left(z^{x}, y^{x}, x,[y, z], y, z\right)$ or $\left(z, y,[y, z], x, y^{x}, z^{x}\right)$.

In case (iii), we apply move (2.9) of [Zim87] to the first alternative and thus reduce the sequence to

$$
\left(z, x y, x y, z, x^{z}, 1\right) .
$$

To the second alternative we have to apply moves (2.1) and a 'mirror move' $\left(2.3^{\prime}\right)$ corresponding to $(2.3)$ of [Zim87] ${ }^{2}$, where the roles of $a_{i}$ and $b_{i+1}$, respectively $a_{i+1}$ and $b_{i}$, are interchanged; more specifically, $\left(2.3^{\prime}\right)$ is

$$
\begin{aligned}
a_{i} & \mapsto a_{i}\left(\left(a_{i} b_{i}^{-1}\right) a_{i+1}^{-1}\left(b_{i} a_{i}^{-1}\right)\right), \\
b_{i} & \mapsto\left(\left(a_{i} b_{i}^{-1}\right) a_{i+1}\left(b_{i} a_{i}^{-1}\right)\right) a_{i+1}^{-1} b_{i}\left(\left(a_{i} b_{i}^{-1}\right) a_{i+1}^{-1}\left(b_{i} a_{i}^{-1}\right)\right), \\
a_{i+1} & \mapsto\left(a_{i} b_{i}^{-1}\right) a_{i+1}\left(b_{i} a_{i}^{-1}\right), \\
b_{i+1} & \mapsto b_{i+1} b_{i} a_{i}^{-1},
\end{aligned}
$$

thus reducing the sequence to $\left(1, x^{z}, z, x y, x y, z\right)$.

In case (iv), we apply move (2.10) of [Zim87], respectively its 'mirror move', and reduce the sequences to

$$
\left(z^{x}, y^{x}, y^{x}, z^{x}, x, 1\right), \quad \text { respectively } \quad\left(1, x, z^{x}, y^{x}, y^{x}, z^{x}\right) .
$$

Now we proceed as in step 1 for the 4-tuples of consecutive elements of type $\left(u^{\prime}, u, u, u^{\prime}\right)$, and we apply Lemma 2.6 of [Zim87] with moves of type (2.1) for the pairs of consecutive elements of type $(x, 1),(1, x)$, and $(x, x)$.

Hence we have shown the stable equivalence of $w^{\prime}$ with

$$
\left(u_{1}, u_{1}^{\prime}, \ldots, u_{g^{\prime}}, u_{g^{\prime}}^{\prime}, z_{g^{\prime}+1}, z_{g^{\prime}+1}^{\prime}, \ldots, z_{k_{1}}, z_{k_{1}}^{\prime}, z_{k_{1}}^{\prime}, z_{k_{1}}, \ldots, z_{g^{\prime}+1}^{\prime}, z_{g^{\prime}+1}\right) .
$$

\footnotetext{
${ }^{2}$ The reference contains a misprint: the first transformation in (2.3) should read $a_{i} \rightarrow a_{i} a_{i+1} b_{i+1}^{-1}$.
} 


\section{F. Catanese, M. Lönne and F. Perroni}

Again, as in step 1 we can get rid of 4-tuples of consecutive elements of type $\left(u^{\prime}, u, u, u^{\prime}\right)$, and we obtain the stable equivalence of $w^{\prime}$ with

$$
w_{\text {et }}=\left(u_{1}, v_{1}, \ldots, u_{g^{\prime}}, v_{g^{\prime}}\right) .
$$

We conclude that the two Hurwitz generating systems $v$ and $w$ are stably equivalent.

We prove Theorem 3.5 by showing that $v$ and $w$ are stably equivalent to Hurwitz generating systems for which the hypotheses of Proposition 4.1 are satisfied. This is achieved through two reduction steps: first we prove that, after stabilization, we may assume $v_{i}=w_{i}$ for $i=1, \ldots, d$ and $G=\left\langle v_{d+1}, \ldots, v_{d+2 g^{\prime}}\right\rangle=\left\langle w_{d+1}, \ldots, w_{d+2 g^{\prime}}\right\rangle$; then we shall stabilize further to obtain $N=0$ in $(4.2)$.

Step 1: Reduction to the case $v_{i}=w_{i}(i=1, \ldots, d)$ and $G=\left\langle v_{d+1}, \ldots, v_{d+2 g^{\prime}}\right\rangle=$ $\left\langle w_{d+1}, \ldots, w_{d+2 g^{\prime}}\right\rangle$

Proposition 4.2. Let

$$
v=\left(c_{1}, \ldots, c_{d} ; a_{1}, b_{1}, \ldots, a_{g^{\prime}}, b_{g^{\prime}}\right) \in \operatorname{HS}\left(G ; g^{\prime}, d\right)
$$

be a Hurwitz generating system, and let $g_{1}, \ldots, g_{d} \in G$. Set $c_{i}^{\prime}=g_{i} c_{i} g_{i}^{-1}$. Then, there exists a $\varphi \in \widehat{\operatorname{Map}}\left(g^{\prime}+d, d\right)$ such that

$$
\varphi \cdot v^{d}=\left(c_{1}^{\prime}, \ldots, c_{d}^{\prime} ; \lambda_{1}, \mu_{1}, \ldots, \lambda_{d}, \mu_{d}, a_{1}, b_{1}, \ldots, a_{g^{\prime}}, b_{g^{\prime}}\right) .
$$

Here $v^{d}$ is obtained from $v$ by adding $d$ handles with trivial monodromies; that is, it is the $d$-stabilization of $v$. Precise formulas for the $\lambda$ and $\mu$ are given below.

We use the following result.

Lemma 4.3. For any

$$
\left(c_{1}, \ldots, c_{d} ; a_{1}, b_{1}, \ldots, a_{g^{\prime}}, b_{g^{\prime}}\right) \in \operatorname{HS}\left(G ; g^{\prime}, d\right)
$$

and for any $x \in G$, we have

$$
\left(c_{1}, \ldots, c_{d} ; 1,1, a_{1}, b_{1}, \ldots, a_{g^{\prime}}, b_{g^{\prime}}\right) \approx\left(c_{1}, \ldots, c_{d} ; x, 1, a_{1}, b_{1}, \ldots, a_{g^{\prime}}, b_{g^{\prime}}\right),
$$

where $\approx$ means that the two vectors are in the same $\widetilde{\operatorname{Map}}\left(g^{\prime}, d\right)$-orbit.

Proof. A direct computation shows that any automorphism $\varphi$ of the form (2.1)-(2.5) in [Zim87] is induced by a transformation $\tilde{\varphi} \in \widetilde{\operatorname{Map}}\left(g^{\prime}, d\right)$ such that $\tilde{\varphi}\left(\gamma_{i}\right)=\gamma_{i}$ for $i=1, \ldots, d$ (cf. step 1 in the proof of Proposition 4.1).

By the method of [Zim87, Lemma 2.6 $]^{3}$, it is true that for any $x \in\left\langle a_{1}, \ldots, b_{g^{\prime}}\right\rangle$ and any $y \in G$,

$$
\left(c_{1}, \ldots, c_{d} ; y, 1, a_{1}, b_{1}, \ldots, a_{g^{\prime}}, b_{g^{\prime}}\right) \approx\left(c_{1}, \ldots, c_{d} ; x y, 1, a_{1}, b_{1}, \ldots, a_{g^{\prime}}, b_{g^{\prime}}\right) .
$$

Therefore it suffices to prove

$$
\left(c_{1}, \ldots, c_{d} ; x, 1, a_{1}, b_{1}, \ldots, a_{g^{\prime}}, b_{g^{\prime}}\right) \approx\left(c_{1}, \ldots, c_{d} ; c_{i} x, 1, a_{1}, b_{1}, \ldots, a_{g^{\prime}}, b_{g^{\prime}}\right)
$$

for any $i=1, \ldots, d$ and any $x$. Using the braid group, it is enough to prove this equivalence when $i=d$ : the result follows as a direct consequence of [CLP15, Proposition A.2(i)] with $\ell=1$

${ }^{3}$ With the method of Zimmermann one would obtain $y x$ instead of $x y$. However, using the moves (2.1) one sees that $(z, 1) \sim\left(z^{-1}, 1\right)$ for all $z$, hence $(y, 1) \sim\left(y^{-1}, 1\right) \sim\left(y^{-1} x^{-1}, 1\right) \sim(x y, 1)$. 


\section{Genus STABILIZATION FOR MODULI OF CURVE-SYMMETRIES}

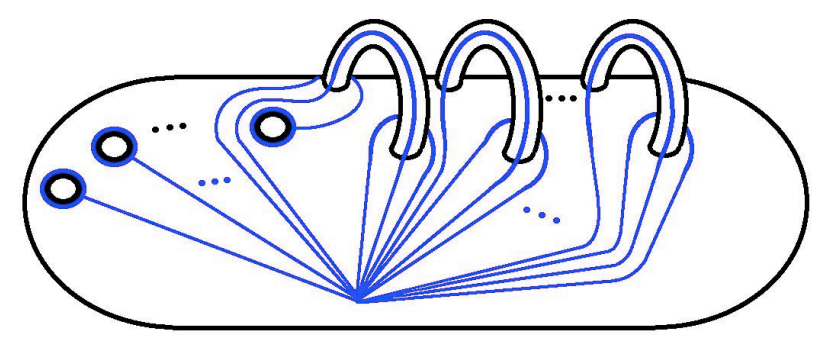

FIGURE 2.

(see Figure 2), yielding the following useful transformation which leaves all the components of the Hurwitz vector unchanged except for

$$
v_{d+1} \mapsto v_{d} v_{d+1}, \quad v_{d} \mapsto g v_{d} g^{-1}, \quad \text { where } g:=\left(v_{d} v_{d+1} v_{d+2} v_{d+1}^{-1}\right) .
$$

In our case $v_{d}=c_{d}, v_{d+1}=x$, and $v_{d+2}=1$, so

$$
v_{d+1}=x \mapsto c_{d} x, \quad v_{d}=c_{d} \mapsto c_{d} .
$$

Proof of Proposition 4.2. The automorphism $\varphi$ is the composition of the mapping classes corresponding to steps 2-7 below. Set $h_{1}=c_{1}^{-1} g_{1}$ and perform the following operations:

1. Add a trivial handle to $v$, obtaining $\left(c_{1}, \ldots, c_{d} ; 1,1, a_{1}, b_{1}, \ldots, a_{g^{\prime}}, b_{g^{\prime}}\right)$.

2. Bring $c_{1}$ to the $d$ th position using the braid group:

$$
\left(c_{1}, \ldots, c_{d} ; 1,1, a_{1}, b_{1}, \ldots, a_{g^{\prime}}, b_{g^{\prime}}\right) \approx\left(c_{1} c_{2} c_{1}^{-1}, \ldots, c_{1} ; 1,1, a_{1}, b_{1}, \ldots, a_{g^{\prime}}, b_{g^{\prime}}\right) .
$$

3. Apply Lemma 4.3 with $x=h_{1}$ :

$$
\left(c_{1} c_{2} c_{1}^{-1}, \ldots, c_{1} ; 1,1, a_{1}, b_{1}, \ldots, a_{g^{\prime}}, b_{g^{\prime}}\right) \approx\left(c_{1} c_{2} c_{1}^{-1}, \ldots, c_{1} ; h_{1}, 1, a_{1}, b_{1}, \ldots, a_{g^{\prime}}, b_{g^{\prime}}\right) .
$$

4. Change $h_{1}, 1$ to $h_{1}, h_{1}$ according to the automorphism of [Zim87, 2.1.b)].

5. Apply [CLP15, Proposition A.2(i)] again with $\ell=1$ (see (4.9) and Figure 2):

$$
\begin{aligned}
\left(c_{1} c_{2} c_{1}^{-1}\right. & \left., \ldots, c_{1} ; h_{1}, h_{1}, a_{1}, b_{1}, \ldots, a_{g^{\prime}}, b_{g^{\prime}}\right) \\
& \approx\left(c_{1} c_{2} c_{1}^{-1}, \ldots,\left(c_{1} h_{1} h_{1} h_{1}^{-1}\right) c_{1}\left(c_{1} h_{1} h_{1} h_{1}^{-1}\right)^{-1} ; c_{1} h_{1}, h_{1}, a_{1}, b_{1}, \ldots, a_{g^{\prime}}, b_{g^{\prime}}\right) \\
& =\left(c_{1} c_{2} c_{1}^{-1}, \ldots, g_{1} c_{1} g_{1}^{-1} ; c_{1} h_{1}, h_{1}, a_{1}, b_{1}, \ldots, a_{g^{\prime}}, b_{g^{\prime}}\right) \\
& =\left(c_{1} c_{2} c_{1}^{-1}, \ldots, c_{1}^{\prime} ; c_{1} h_{1}, h_{1}, a_{1}, b_{1}, \ldots, a_{g^{\prime}}, b_{g^{\prime}}\right) .
\end{aligned}
$$

6. Use the braid group to move the last monodromy to the first position:

$$
\left(c_{1} c_{2} c_{1}^{-1}, \ldots, c_{1}^{\prime} ; c_{1} h_{1}, h_{1}, a_{1}, b_{1}, \ldots, a_{g^{\prime}}, b_{g^{\prime}}\right) \approx\left(c_{1}^{\prime}, \overline{c_{2}}, \ldots, \overline{c_{d}} ; c_{1} h_{1}, h_{1}, a_{1}, b_{1}, \ldots, a_{g^{\prime}}, b_{g^{\prime}}\right),
$$

where $\overline{c_{i}}$ is a conjugate of $c_{i}$ for all $i$.

7. Repeat the steps above for $\overline{c_{2}}$, with $h_{2}={\overline{c_{2}}}^{-1} \overline{g_{2}}, c_{2}^{\prime}=\overline{g_{2}} \overline{c_{2}}{\overline{g_{2}}}^{-1}$, and so on.

Remark 4.4. The condition $G=\left\langle v_{d+1}, \ldots, v_{d+2 g^{\prime}}\right\rangle=\left\langle w_{d+1}, \ldots, w_{d+2 g^{\prime}}\right\rangle$ in Proposition 4.1 can be achieved by using Lemma 4.3.

\section{Step 2: Reduction to the case $N=0$}

Let $v$ and $w$ be Hurwitz generating systems as in the beginning of the section. By step 1, we may assume $v_{i}=w_{i}$ for $i=1, \ldots, d$ and $G=\left\langle v_{d+1}, \ldots, v_{d+2 g^{\prime}}\right\rangle=\left\langle w_{d+1}, \ldots, w_{d+2 g^{\prime}}\right\rangle$. By hypothesis 


\section{F. Catanese, M. Lönne and F. Perroni}

we have

$$
\operatorname{ev}(\hat{w}) \equiv \operatorname{ev}(\hat{v}) \prod_{\ell=1}^{N}\left(\widehat{x}_{\ell} \widehat{y} \ell_{\ell} \widehat{z}^{-1} \widehat{y}^{-1}\right)^{\sigma_{\ell}} \quad(\bmod [F, R]),
$$

where $x_{\ell} y_{\ell} z_{\ell}^{-1} y_{\ell}^{-1}=1, \sigma_{\ell}= \pm 1$, and $x_{\ell}, z_{\ell} \in \Gamma$.

The main result of this subsection is the following.

Proposition 4.5. Let $v, w \in \operatorname{HS}\left(G ; g^{\prime}, d\right)$ be Hurwitz generating systems with $\nu(v)=\nu(w)$ and $\varepsilon(v)=\varepsilon(w)$. Assume further $v_{i}=w_{i}$ for $i=1, \ldots, d$ and $G=\left\langle v_{d+1}, \ldots, v_{d+2 g^{\prime}}\right\rangle=$ $\left\langle w_{d+1}, \ldots, w_{d+2 g^{\prime}}\right\rangle$. Then there exist an $h \in \mathbb{N}$ and $\varphi, \psi \in \widehat{\operatorname{Map}}\left(g^{\prime}+h, d\right)$ such that

$$
\begin{aligned}
\operatorname{ev}\left(\widehat{\psi \cdot w^{h}}\right) & \equiv \operatorname{ev}\left(\widehat{\varphi \cdot v^{h}}\right) & (\bmod [F, R]) \\
\left(\psi \cdot w^{h}\right)_{i} & =\left(\varphi \cdot v^{h}\right)_{i} \quad & \text { for all } i=1, \ldots, d .
\end{aligned}
$$

To prove Proposition 4.5, we first rewrite $\prod_{\ell=1}^{N}\left(\widehat{x_{\ell}} \widehat{y_{\ell}} \widehat{z} \ell^{-1} \widehat{y} \ell^{-1}\right)^{\sigma_{\ell}}$ in (4.10) as a product of commutators of the form $[\hat{\eta}, \hat{\xi}]$, where $\xi \in \Gamma$ and $[\eta, \xi]=1$. To achieve this, we use the following identities.

Lemma 4.6. Let $x, y, z, y_{1}, z_{1} \in G$ be such that

$$
x y z^{-1} y^{-1}=z y_{1} z_{1}^{-1} y_{1}^{-1}=1 .
$$

Then the following congruences hold, where as usual $\hat{x}, \hat{y}, \hat{z}, \widehat{y_{1}}, \widehat{z_{1}} \in F$ are the tautological lifts of $x, y, z, y_{1}, z_{1}$ :

(i) $\widehat{x} \widehat{y} \widehat{z}^{-1} \widehat{y}^{-1} \equiv \widehat{y}^{-1} \widehat{x} \widehat{y} \widehat{z}^{-1}(\bmod [F, R])$;

(ii) $\left(\widehat{x} \widehat{y}^{-1} \widehat{y}^{-1}\right)^{-1}=\widehat{y} \widetilde{z} \widehat{y}^{-1} \widehat{x}^{-1} \equiv \widehat{z} \widehat{y}^{-1} \widehat{x}^{-1} \widehat{y}(\bmod [F, R])$;

(iii) $\left(\hat{x} \hat{y} \hat{z}^{-1} \hat{y}^{-1}\right)\left(\hat{z} \widehat{y}_{1} \widehat{z}_{1}^{-1} \widehat{y}_{1}^{-1}\right) \equiv \hat{x} \widehat{y y}_{1} \widehat{z}_{1}^{-1}{\widehat{y y_{1}}}^{-1}(\bmod [F, R])$;

(iv) $\hat{x} \hat{y}^{\sigma} \hat{z}^{-1} \hat{y}^{-\sigma} \equiv \hat{x} \widehat{y^{\sigma}} \hat{z}^{-1} \widehat{y}^{\sigma}-1(\bmod [F, R])$, where $\sigma= \pm 1$.

Proof. Congruences (i) and (ii) follow from the fact that if $r \in R$, then

$$
r \equiv \widehat{y}^{-1} r \widehat{y} \quad(\bmod [F, R]) .
$$

For congruence (iii), notice that using congruence (i), we have

$$
\left(\hat{x} \hat{y} \hat{z}^{-1} \hat{y}^{-1}\right)\left(\hat{z} \widehat{y}_{1} \widehat{z}_{1}^{-1} \widehat{y}_{1}^{-1}\right) \equiv\left(\hat{y}^{-1} \hat{x} \hat{y} \hat{z}^{-1}\right)\left(\hat{z} \widehat{y}_{1} \widehat{z}_{1}^{-1}{\widehat{y_{1}}}^{-1}\right)=\hat{y}^{-1} \hat{x} \hat{y}{\widehat{y_{1}}}_{z_{1}}^{-1}{\widehat{y_{1}}}^{-1} \quad(\bmod [F, R]) .
$$

Moreover, this element is in $R$, since it is congruent to a product of two elements in $R$ modulo $[F, R]$. Hence we have by the usual token

$$
\hat{y}^{-1} \hat{x} \hat{y}{\widehat{y_{1}}}_{z_{1}}^{-1}{\widehat{y_{1}}}^{-1} \equiv \hat{x} \hat{y} \widehat{y}_{1}{\widehat{z_{1}}}^{-1}{\widehat{y_{1}}}^{-1} \hat{y}^{-1} \quad(\bmod [F, R]) ;
$$

in fact, the right-hand side is just the conjugate of the left-hand side by $\widehat{y}$. Finally,

$$
\left(\hat{x} \hat{y}{\widehat{y_{1}}}_{z_{1}}^{-1}{\widehat{y_{1}}}^{-1} \hat{y}^{-1}\right)\left(\hat{x} \widehat{y y}_{1} \widehat{z}_{1}^{-1}{\widehat{y y_{1}}}^{-1}\right)^{-1}=\left(\hat{x} \hat{y} \widehat{y}_{1}\right)\left[\widehat{z}_{1}^{-1}, \widehat{y}_{1}^{-1} \hat{y}^{-1} \widehat{y y_{1}}\right]\left(\hat{x} \hat{y} \widehat{y}_{1}\right)^{-1} \in[F, R] .
$$

For congruence (iv) we use that $[F, R]$ is a normal subgroup of $F$. We have

$$
\left(\hat{x} \hat{y}^{\sigma} \hat{z}^{-1} \hat{y}^{-\sigma}\right)\left(\hat{x} \widehat{y^{\sigma}} \hat{z}^{-1} \widehat{y}^{\sigma}-1\right)^{-1}=\left(\hat{x} \hat{y}^{\sigma}\right)\left[\hat{z}^{-1}, \hat{y}^{-\sigma} \widehat{y^{\sigma}}\right]\left(\hat{x} \hat{y}^{\sigma}\right)^{-1} \in[F, R] .
$$

Lemma 4.7. Let $v, w$ be as in Proposition 4.5. Then there exist $M \in \mathbb{N}$ and, for $m=1, \ldots, M$, elements $\xi_{m} \in \Gamma$ and $\eta_{m} \in G$ with $\left[\xi_{m}, \eta_{m}\right]=1$, such that

$$
\operatorname{ev}(\hat{w}) \equiv \operatorname{ev}(\hat{v}) \prod_{m=1}^{M}\left[\widehat{\xi_{m}}, \widehat{\eta_{m}}\right] \quad(\bmod [F, R]) .
$$




\section{Genus STABILIZATION FOR MODUli OF CURVE-SYMMETRIES}

Proof. Using Lemma 4.6(ii) and (iv), rewrite (4.10) as

$$
\operatorname{ev}(\hat{w}) \equiv \operatorname{ev}(\hat{v}) \prod_{\ell=1}^{N} \widehat{a_{\ell}} \widehat{b}_{\ell} \widehat{c}_{\ell}^{-1} \widehat{b}_{\ell}^{-1} \quad(\bmod [F, R])
$$

where $a_{\ell}=x_{\ell}, b_{\ell}=y_{\ell}, c_{\ell}=z_{\ell}$ if $\sigma_{\ell}=1$, and $a_{\ell}=z_{\ell}, b_{\ell}=y_{\ell}^{-1}, c_{\ell}=x_{\ell}$ if $\sigma_{\ell}=-1$.

Consider the image of (4.11) in the abelianized group $F^{a b}$. Since $v_{i}=w_{i}$ for $i=1, \ldots, d$, we get

$$
\prod_{\ell=1}^{N} \widehat{a_{\ell}}{\widehat{c_{\ell}}}^{-1}=1 \quad(\bmod [F, F]) .
$$

Hence there exists a permutation $\tau \in \mathfrak{S}_{N}$ such that $c_{\ell}=a_{\tau(\ell)}$ for every $\ell=1, \ldots, N$.

Let us treat first the case where $\tau$ is a cycle of length $N$. In this case the set $\left\{a_{\tau^{k}(1)} \mid k \in \mathbb{N}\right\}$ equals $\left\{a_{1}, \ldots, a_{N}\right\}$ and, since the product of the factors $(\bmod [F, R])$ is independent of the order,

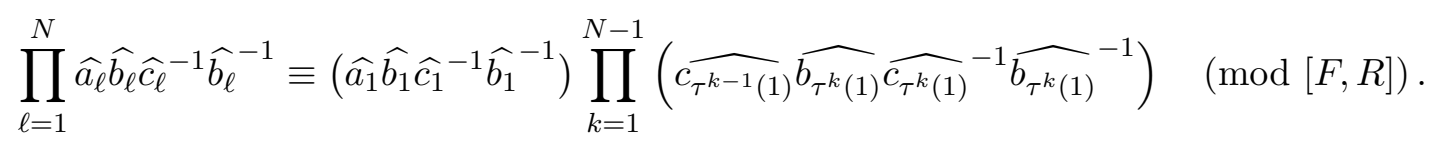

Setting $\xi_{1}:=a_{1}$ and $\eta_{1}:=\prod_{k=0}^{N-1} b_{\tau^{k}(1)}$, we obtain

$$
\prod_{\ell=1}^{N} \widehat{a_{\ell}} \widehat{b}_{\ell}{\widehat{c_{\ell}}}^{-1} \widehat{b}_{\ell}^{-1} \equiv\left[\widehat{\xi_{1}}, \widehat{\eta}_{1}\right] \quad(\bmod [F, R])
$$

where the equivalence follows from Lemma 4.6(iii). Since the left-hand side of this equivalence is in $R$, it follows that $\left[\xi_{1}, \eta_{1}\right]=1$.

The general case, where $\tau$ is a product of cycles of length less than $N$, follows by induction.

To complete the proof of Proposition 4.5, we need to know how ev $(\hat{v})$ changes under the action of the mapping class group, modulo $[F, R]$. Notice in fact that $\operatorname{ev}(\hat{v})$ is $\widetilde{\operatorname{Map}}\left(g^{\prime}, d\right)$-invariant only modulo $R_{\Gamma}$.

Lemma 4.8. Let $v \in \operatorname{HS}\left(G ; g^{\prime}, d\right)$ and let $\varphi \in \widetilde{\operatorname{Map}}\left(g^{\prime}, d\right)$. Then we have

(i) $\operatorname{ev}(\widehat{\varphi \cdot v}) \equiv \mathrm{ev}(\hat{v})(\bmod [F, R])$ if $\varphi\left(\gamma_{i}\right)=\gamma_{i}$ for $i=1, \ldots, d$;

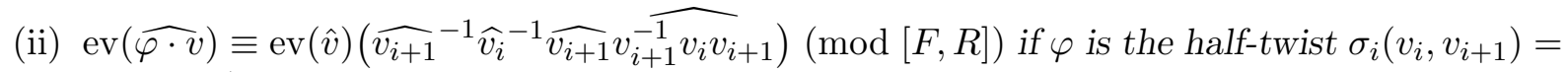
$\left(v_{i+1}, v_{i+1}^{-1} v_{i} v_{i+1}\right)$

(iii) $\operatorname{ev}(\widehat{\varphi \cdot v}) \equiv \operatorname{ev}(\hat{v})\left(\widehat{g} \widehat{v}_{d}^{-1} \widehat{g}^{-1} \widehat{g v_{d} g^{-1}}\right)(\bmod [F, R])$ if $\varphi$ is as in (4.9) ([CLP15, Proposition A.2(i)] with $\ell=1$ ), and then $g=v_{d} v_{d+1} v_{d+2} v_{d+1}^{-1}$.

Proof. The proof is similar to that of [CLP15, Proposition 3.6] (invariance of the $\varepsilon$-invariant under $\left.\operatorname{Map}\left(g^{\prime}, d\right)\right)$.

Let $\alpha: F /[F, R] \rightarrow G$ be the morphism $\hat{g} \mapsto g$. Then $\operatorname{ker}(\alpha)=R /[F, R]$ is central in $F /[F, R]$. We have

$$
\alpha\left((\widehat{\varphi \cdot v})_{i}\right)=(\varphi \cdot v)_{i}=\alpha\left((\varphi \cdot \hat{v})_{i}\right) \quad \text { for } i=1, \ldots, d+2 g^{\prime} .
$$

Hence, there are $\zeta_{i} \in R /[F, R]$ such that

$$
(\widehat{\varphi \cdot v})_{i}=(\varphi \cdot \hat{v})_{i} \cdot \zeta_{i} \quad \text { for } i=1, \ldots, d+2 g^{\prime} .
$$




\section{F. Catanese, M. Lönne and F. Perroni}

Since $R /[F, R] \leqslant F /[F, R]$ is central, we can replace $(\widehat{\varphi \cdot v})_{i}$ with $(\varphi \cdot \hat{v})_{i}$ for $i=d+1, \ldots, d+2 g^{\prime}$ in the expression for ev $(\widehat{\varphi \cdot v})$. This is enough to prove the claim if $\varphi$ acts as the identity on $\gamma_{i}$ for $i=1, \ldots, d$. Indeed, in this case, we have

$$
\operatorname{ev}(\widehat{\varphi \cdot v})=\operatorname{ev}(\varphi \cdot \hat{v})=\operatorname{ev}(\hat{v})
$$

by the fact that evaluation is invariant under mapping classes. This proves statement (i).

Statement (ii) follows by a direct computation.

Finally, consider the case where $\varphi$ is as defined in (4.9) ([CLP15, Proposition A.2(i)] with $\ell=1)$. We have $(\varphi \cdot v)_{d}=g v_{d} g^{-1}$ and $(\varphi \cdot v)_{i}=v_{i}$ for $i=1, \ldots, d-1$, where $g \in G$ is given above. Let $x=\widehat{v_{d}} \widehat{v_{d+1}} \widehat{v_{d+2}} \widehat{v_{d+1}}-1 \in F /[F, R]$; then $(\varphi \cdot \hat{v})_{d}=x \widehat{v_{d}} x^{-1}$. Since $\alpha(x)=g$, there is an $\eta \in R /[F, R]$ (in particular, $\eta$ is central) such that $x=\widehat{g} \eta$. It follows that

$$
(\varphi \cdot \hat{v})_{d}=(\widehat{g} \eta) \widehat{v_{d}}(\widehat{g} \eta)^{-1}=\widehat{g} \widehat{v}_{d} \widehat{g}^{-1}
$$

Now, notice that

$$
\begin{aligned}
(\widehat{\varphi \cdot v})_{d} & =\widehat{g v_{d} g^{-1}}=\widehat{g} \widehat{v}_{d} \widehat{g}^{-1}\left(\widehat{g} \widehat{v}_{d}^{-1} \widehat{g}^{-1} \widehat{g v_{d} g^{-1}}\right) \\
& =(\varphi \cdot \hat{v})_{d}\left(\widehat{g} \widehat{v}_{d}^{-1} \widehat{g}^{-1} \widehat{g v_{d} g^{-1}}\right)
\end{aligned}
$$

from which the claim follows.

Proof of Proposition 4.5. Let $M \in \mathbb{N}, \xi_{m} \in \Gamma$, and $\eta_{m} \in G$ be as in Lemma 4.7. Then

$$
\operatorname{ev}(\hat{w}) \equiv \operatorname{ev}(\hat{v}) \prod_{m=1}^{M}\left[\widehat{\xi_{m}}, \widehat{\eta_{m}}\right] \quad(\bmod [F, R]) .
$$

Since $\xi_{1} \in \Gamma$, there exists a $k \in\{1, \ldots, d\}$ such that $v_{k}=w_{k}$ is conjugate to $\xi_{1}$. Without loss of generality, we assume $k=d$; otherwise we move $v_{k}$ and $w_{k}$ simultaneously to the $d$ th position using elementary braids.

Argueing as in Proposition 4.2, there exist $\varphi_{1}^{\prime}, \varphi_{2}^{\prime} \in \widetilde{\operatorname{Map}}\left(g^{\prime}+1, d\right)$ such that

$$
\begin{aligned}
\operatorname{ev}\left(\widehat{\varphi_{2}^{\prime} \cdot w^{1}}\right) & \equiv \operatorname{ev}\left(\widehat{\varphi_{1}^{\prime} \cdot v^{1}}\right) \prod_{m=1}^{M}\left[\widehat{\xi_{m}}, \widehat{\eta_{m}}\right] \quad(\bmod [F, R]), \\
\left(\varphi_{2}^{\prime} \cdot w^{1}\right)_{i} & =\left(\varphi_{1}^{\prime} \cdot v^{1}\right)_{i} \quad \text { for } i=1, \ldots, d, \\
\left(\varphi_{2}^{\prime} \cdot w^{1}\right)_{d} & =\xi_{1} .
\end{aligned}
$$

Equation (4.13) holds true because of (4.12) and Lemmas 4.8 and 4.3. Moreover, there also exists a $\varphi_{1} \in \widetilde{\operatorname{Map}}\left(g^{\prime}+2, d\right)$ such that $\varphi_{1} \cdot v^{2}=\left(\varphi_{1}^{\prime} \cdot v^{1}\right)^{1}$, the vector obtained by adding one handle with trivial monodromies to $\varphi_{1}^{\prime} \cdot v^{1}$.

By the argument of the proof of Lemma 4.3 , this vector is $\widetilde{\operatorname{Map}}\left(g^{\prime}+2, d\right)$-equivalent to

$$
\left(v_{1}, \ldots, v_{d-1}, \xi_{1} ; \eta_{1}, 1,\left(\varphi_{1}^{\prime} \cdot v^{1}\right)_{d+1},\left(\varphi_{1}^{\prime} \cdot v^{1}\right)_{d+2}, v_{d+1}, \ldots, v_{d+2 g^{\prime}}\right)
$$

using only transformations as in Lemma 4.8(i). Here we use the assumption that the $v_{j}$ for $j>d$ generate $G$.

Using the automorphisms of [Zim87, 2.1.a) and b)], we see that the vector is further equivalent to

$$
\left(v_{1}, \ldots, v_{d-1}, \xi_{1} ; 1, \eta_{1},\left(\varphi_{1}^{\prime} \cdot v^{1}\right)_{d+1},\left(\varphi_{1}^{\prime} \cdot v^{1}\right)_{d+2}, v_{d+1}, \ldots, v_{d+2 g^{\prime}}\right)
$$


Now, by (4.9) ([CLP15, Proposition A.2(i)] with $\ell=1)$ there exists a $\varphi_{2} \in \widetilde{\operatorname{Map}}\left(g^{\prime}+2, d\right)$ such that

$$
\begin{aligned}
\varphi_{2} \cdot\left(\varphi_{1} \cdot v^{2}\right) & =\left(v_{1}, \ldots, v_{d-1}, \xi_{1} \eta_{1} \xi_{1} \eta_{1}^{-1} \xi_{1}^{-1} ; \xi_{1}, \eta_{1},\left(\varphi_{1}^{\prime} \cdot v^{1}\right)_{d+1},\left(\varphi_{1}^{\prime} \cdot v^{1}\right)_{d+2}, v_{d+1}, \ldots, v_{d+2 g^{\prime}}\right) \\
& =\left(v_{1}, \ldots, v_{d-1}, \xi_{1} ; \xi_{1}, \eta_{1},\left(\varphi_{1}^{\prime} \cdot v^{1}\right)_{d+1},\left(\varphi_{1}^{\prime} \cdot v^{1}\right)_{d+2}, v_{d+1}, \ldots, v_{d+2 g^{\prime}}\right)
\end{aligned}
$$

since $\left[\xi_{1}, \eta_{1}\right]=1$ according to Lemma 4.7 . The same property implies

$$
\mathrm{ev}\left(\varphi_{2} \cdot \widehat{\varphi_{1}} \cdot v^{2}\right)=\operatorname{ev}\left(\widehat{\varphi_{1} \cdot v^{2}}\right)\left[\widehat{\xi_{1}}, \widehat{\eta_{1}}\right] .
$$

This proves the desired assertion if $M=1$. The general case, where $M>1$, is proven inductively along the same lines.

\section{ACKNOWLEDGEMENTS}

We thank the referee for a very careful reading of the first version of this article, for pointing out the need of adjustments for some arguments by Zimmermann in [Zim87], and for useful suggestions and remarks.

\section{REFERENCES}

ACG11 E. Arbarello, M. Cornalba, and P.A. Griffiths, Geometry of algebraic curves. Vol. II, Grundlehren Math. Wiss., vol. 268 (Springer, Heidelberg, 2011); http://dx.doi.org/10.1007/ 978-3-540-69392-5.

BC97 I. Bauer and F. Catanese, Generic lemniscates of algebraic functions, Math. Ann. 307 (1997), no. 3, 417-444; http://dx.doi.org/10.1007/s002080050042.

BF86 R. Biggers and M. Fried, Irreducibility of moduli spaces of cyclic unramified covers of genus $g$ curves, Trans. Amer. Math. Soc. 295 (1986), no. 1, 59-70; http://dx.doi.org/10.2307/ 2000145.

Bir69 J.S. Birman, Mapping class groups and their relationship to braid groups, Comm. Pure Appl. Math. 22 (1969), 213-238; http://dx.doi.org/10.1002/cpa.3160220206.

Cat88 F. Catanese, Moduli of algebraic surfaces, Theory of Moduli (Montecatini Terme, 1985), Lecture Notes in Math., vol. 1337 (Springer, Berlin, 1988), 1-83; http://dx.doi.org/10.1007/ $\mathrm{BFb} 0082806$.

Cat00 , Fibred surfaces, varieties isogenous to a product and related moduli spaces, Amer. J. Math. 122 (2000), no. 1, 1-44; http://www. jstor.org/stable/25098976.

Cat08 Differentiable and deformation type of algebraic surfaces, real and symplectic structures, in Symplectic 4-manifolds and algebraic surfaces, Lecture Notes in Math., vol. 1938 (Springer, Berlin, 2008), 55-167; http://dx.doi.org/10.1007/978-3-540-78279-7_2.

Cat12 Irreducibility of the space of cyclic covers of algebraic curves of fixed numerical type and the irreducible components of $\operatorname{Sing}\left(\overline{\mathfrak{M}_{g}}\right)$, in Advances in Geometric Analysis, Adv. Lect. Math. (ALM), vol. 21 (Int. Press, Somerville, MA, 2012), 281-306.

Cle73 A. Clebsch, Zur Theorie der Riemann'schen Flächen, Math. Ann. 6 (1873), no. 2, 216-230; http://dx.doi.org/10.1007/BF01443193.

CLP11 F. Catanese, M. Lönne, and F. Perroni, Irreducibility of the space of dihedral covers of the projective line of a given numerical type, Atti Accad. Naz. Lincei Cl. Sci. Fis. Mat. Natur. Rend. Lincei (9) Mat. Appl. 22 (2011), no. 3, 291-309; http://dx.doi.org/10.4171/RLM/601, arXiv:1102.0490. 


\section{F. Catanese, M. Lönne and F. Perroni}

CLP15

The irreducible components of the moduli space of dihedral covers of algebraic curves, Groups Geom. Dyn. 9 (2015), no. 4, 1185-1229; http://dx.doi.org/10.4171/GGD/338, arXiv:1206.5498.

Cor87 M. Cornalba, On the locus of curves with automorphisms, Ann. Mat. Pura Appl. (4) 149 (1987), no. 1, 135-151; http://dx.doi.org/10.1007/BF01773930.

Cor08_, Erratum: "On the locus of curves with automorphisms" [Ann. Mat. Pura Appl. (4) 149 (1987), 135-151], Ann. Mat. Pura Appl. (4) 187 (2008), no. 1, 185-186; http://dx.doi.org/ 10.1007/s10231-006-0031-0.

DM69 P. Deligne and D. Mumford, The irreducibility of the space of curves of given genus, Publ. Math. Inst. Hautes Études Sci. 36 (1969), no. 1, 75-109; http://dx.doi.org/10.1007/BF02684599.

DT06 N. M. Dunfield and W.P. Thurston, Finite covers of random 3-manifolds, Invent. Math. 166 (2006), no. 3, 457-521; http://dx.doi.org/10.1007/s00222-006-0001-6.

Edm82 A. L. Edmonds, Surface symmetry I, Michigan Math. J. 29 (1982), no. 2, 171-183; http://dx. doi.org/10.1307/mmj/1029002670.

Edm83 Surface symmetry II, Michigan Math. J. 30 (1983), no. 2, 143-154; http://dx.doi. org/10.1307/mmj/1029002844.

Ful69 W. Fulton, Hurwitz schemes and irreducibility of moduli of algebraic curves, Ann. of Math. (2) 90 (1969), no. 3, 542-575; http://dx.doi.org/10.2307/1970748.

FV91 M. D. Fried and H. Völklein, The inverse Galois problem and rational points on moduli spaces, Math. Ann. 290 (1991), no. 4, 771-800; http://dx.doi.org/10.1007/BF01459271.

GHS02 T. Graber, J. Harris, and J. Starr, A note on hurwitz schemes of covers of a positive genus curve, arXiv:math.AG/0205056.

Har85 J.L. Harer, Stability of the homology of the mapping class groups of orientable surfaces, Ann. of Math. (2) 121 (1985), no. 2, 215-249; http://dx.doi.org/10.2307/1971172.

Hop42 H. Hopf, Fundamentalgruppe und zweite Bettische Gruppe, Comment. Math. Helv. 14 (1942), no. 1, 257-309; http://dx.doi.org/10.1007/BF02565622.

Hur91 A. Hurwitz, Ueber Riemann'sche Flächen mit gegebenen Verzweigungspunkten, Math. Ann. 39 (1891), no. 1, 1-60; http://dx.doi.org/10.1007/BF01199469.

Kan05 V. Kanev, Irreducibility of hurwitz spaces, arXiv:math.AG/0509154.

Kan06__ Hurwitz spaces of Galois coverings of $\mathbb{P}^{1}$, whose Galois groups are Weyl groups, J. Algebra 305 (2006), no. 1, 442-456; http://dx.doi.org/10.1016/j.jalgebra.2006.01.008.

Klu88 P. Kluitmann, Hurwitz action and finite quotients of braid groups, Braids (Santa Cruz, CA, 1986), Contemp. Math., vol. 78 (Amer. Math. Soc., Providence, RI, 1988), 299-325; http://dx. doi.org/10.1090/conm/078/975086.

Liv85 C. Livingston, Stabilizing surface symmetries, Michigan Math. J. 32 (1985), no. 2, 249-255; http://dx.doi.org/10.1307/mmj/1029003192.

Mas91 W.S. Massey, A basic course in algebraic topology, Grad. Texts in Math., vol. 127 (SpringerVerlag, New York, 1991).

Mil52 C. Miller, The second homology group of a group; relations among commutators, Proc. Amer. Math. Soc. 3 (1952), no. 4, 588-595; http://dx.doi.org/10.1090/ S0002-9939-1952-0049191-5.

Nie37 J. Nielsen, Die Struktur periodischer Transformationen von Flächen, Danske Vidensk. Selsk. Math.-Fys. Medd. 15 (1937), no. 1, 1-77.

Sia09 C. Sia, Hurwitz equivalence in tuples of dihedral groups, dicyclic groups, and semidihedral groups, Electron. J. Combin. 16 (2009), no. 1, Research Paper 95; http://www.combinatorics.org/ Volume_16/Abstracts/v16i1r95.html.

Vet06 F. Vetro, Irreducibility of Hurwitz spaces of coverings with one special fiber, Indag. Math. (N.S.) 17 (2006), no. 1, 115-127; http://dx.doi.org/10.1016/S0019-3577(06)80010-8. 


\section{Genus STABILIZATION FOR MODULi OF CURVE-SyMmetries}

Vet07

Irreducibility of Hurwitz spaces of coverings with monodromy groups Weyl groups of type $W\left(B_{d}\right)$, Boll. Unione Mat. Ital. Sez. B Artic. Ric. Mat. (8) 10 (2007), no. 2, 405-431.

Vet08_- Irreducibility of Hurwitz spaces of coverings with one special fiber and monodromy group a Weyl group of type $D_{d}$, Manuscripta Math. 125 (2008), no. 3, 353-368; http://dx.doi.org/ 10.1007/s00229-007-0153-8.

Waj96 B. Wajnryb, Orbits of Hurwitz action for coverings of a sphere with two special fibers, Indag. Math. (N.S.) 7 (1996), no. 4, 549-558; http://dx.doi.org/10.1016/S0019-3577 (97) 89139-2.

Waj99 An elementary approach to the mapping class group of a surface, Geom. Topol. 3 (1999), no. 1, 405-466, http://dx.doi.org/10.2140/gt.1999.3.405.

Whi78 G. W. Whitehead, Elements of homotopy theory, Grad. Texts in Math., vol. 61 (Springer-Verlag, New York - Berlin, 1978); http://dx.doi.org/10.1007/978-1-4612-6318-0.

Zim87 B. Zimmermann, Surfaces and the second homology of a group, Monatsh. Math. 104 (1987), no. 3, 247-253; http://dx.doi.org/10.1007/BF01547955.

Fabrizio Catanese Fabrizio.Catanese@uni-bayreuth.de

Lehrstuhl Mathematik VIII, Mathematisches Institut der Universität Bayreuth, NW II, Universitätsstr. 30, 95447 Bayreuth, Germany

Michael Lönne Michael.Loenne@uni-bayreuth.de

Lehrstuhl Mathematik VIII, Mathematisches Institut der Universität Bayreuth, NW II, Universitätsstr. 30, 95447 Bayreuth, Germany

Fabio Perroni fperroni@units.it

SISSA - International School for Advanced Studies, School of Mathematics, via Bonomea, 265 34136 Trieste, Italy

Current address: Dipartimento di Matematica e Geoscienze, Sezione di Matematica e Informatica, Università di Trieste, Via Valerio 12/1, 34127 Trieste, Italy 